\title{
Inhibition of Extracellular Calcium Influx Results in Enhanced IL-12 Production in LPS-Treated Murine Macrophages by Downregulation of the CaMKK $\beta$-AMPK-SIRT1 Signaling Pathway
}

\author{
Xin Liu, ${ }^{1}$ Ning Wang, ${ }^{1}$ Yuanfeng Zhu, ${ }^{1}$ Yongjun Yang, ${ }^{1}$ Xiaoli Chen, ${ }^{1}$ Shijun Fan, \\ Qian Chen, ${ }^{1}$ Hong Zhou, ${ }^{2}$ and Jiang Zheng ${ }^{1}$ \\ ${ }^{1}$ Medical Research Center, Southwest Hospital, The Third Military Medical University, Chongqing 400038, China \\ ${ }^{2}$ Department of Pharmacology, College of Pharmacy, The Third Military Medical University, Chongqing 400038, China
}

Correspondence should be addressed to Hong Zhou; zhouh64@163.com and Jiang Zheng; zhengj@tmmu.edu.cn

Received 19 January 2016; Revised 19 April 2016; Accepted 27 April 2016

Academic Editor: Soh Yamazaki

Copyright (C) 2016 Xin Liu et al. This is an open access article distributed under the Creative Commons Attribution License, which permits unrestricted use, distribution, and reproduction in any medium, provided the original work is properly cited.

\begin{abstract}
Activated macrophages are the primary sources of IL-12, a key cytokine bridging innate and adaptive immunity. However, macrophages produce low amounts of IL-12 upon stimulation and the underlying regulatory mechanism remains unclear. In this study, we found a new calcium-dependent mechanism that controlled IL-12 production in LPS-treated murine macrophages. First, LPS was demonstrated to induce extracellular calcium entry in murine peritoneal macrophages and inhibition of calcium influx resulted in marked enhancement in IL-12 production. Then, withdrawal of extracellular calcium was found to suppress CaMKK $\beta$ and AMPK activation triggered by LPS while chemical inhibition or genetic knockdown of these two kinases augmented LPS induced IL-12 production. AMPK activation increased the NAD ${ }^{+} / \mathrm{NADH}$ ratio and activated Sirtuin 1 (SIRT1), a NAD ${ }^{+}$-dependent deacetylating enzyme and negative regulator of inflammation. Chemical inhibitor or siRNA of SIRT1 enhanced IL-12 release while its agonist suppressed IL-12 production. Finally, it was found that SIRT1 selectively affected the transcriptional activity of NF- $\kappa$ B which thereby inhibited IL-12 production. Overall, our study demonstrates a new role of transmembrane calcium mobilization in immunity modulation such that inhibition of calcium influx leads to impaired activation of CaMKK $\beta$-AMPK-SIRT1 signaling pathway which lifts restriction on NF- $\kappa \mathrm{B}$ activation and results in enhanced IL-12 production.
\end{abstract}

\section{Introduction}

The recruitment and activation of macrophages in response to microbial products such as lipopolysaccharide (LPS) lead to production of various immune/inflammatory effectors, which are essential for the anti-infectious immune response. Interleukin 12 (IL-12), a $70 \mathrm{kD}$ (p70) heterodimeric cytokine comprised of a p40 and a p35 subunit separately, is mainly produced by activated macrophages and plays a pivotal role in connecting innate immunity with adaptive immunity [1]. The expression of IL-12 is primarily modulated at transcriptional level in macrophages. Indeed, various control elements have been revealed in the expression of inducible p 40 gene. For example, it was found that a sequence in NF- $\kappa$ B (p50/p65 and p50/c-Rel) complexes was functionally essential for modulating the promoter activity of IL-12 p40 in response to several p40-inducing pathogens $[2,3]$. Other regulatory elements, such as C/EBP, ETS-2, and IRF1, have been also implicated in mediating the inducible promoter activity of IL$12[1,4-6]$. However, the upstream signaling events that connect microbial stimulation with downstream transcriptional regulation to control IL-12 expression in macrophages remain largely unknown.

Extracellular calcium influx across the plasma membrane is a major source for the increase of intracellular calcium, which acts as a crucial secondary messenger in regulating macrophage activation, such as phagosomes maturation, activation of NLRP3 inflammasome, and transcriptional control 
of cytokines [7-9]. Microbial components (LPS, etc.) are known to elicit calcium influx in murine macrophages and thereby upregulate the production of mediators like NO and IL-10 [10-12]. However, it has been found that single LPS stimulation is insufficient for the upregulation of IL-12 production and there is a negative relationship between the consequences of extracellular derived calcium increase and the insufficient production of IL-12. For example, ligation of $\mathrm{Fc} \gamma$, complement, or scavenger receptors in macrophage inhibited the induction of IL-12 by LPS. Moreover, further experimental approaches suggested that IL-12 inhibition was due to extracellular calcium influxes that occurred after receptor ligation [13]. In our previous study, we demonstrated that clathrin/dynamin-dependent internalization, a process that interplayed with calcium influx, also resulted in the elevation of IL-12 secretion in murine macrophages [14]. Such results suggest that calcium may activate different intracellular signaling events which may exert an opposite function for IL-12 regulation. However, the precise regulatory mechanisms require further elucidation.

Generally, intracellular calcium binds to calmodulin $(\mathrm{CaM})$, activates CaM kinases, and further regulates transcriptional events in inflammation and immunity. Calcium/ calmodulin dependent protein kinase kinase $\beta(\mathrm{CaMKK} \beta)$ is a key CaM kinase activated by the increased intracellular calcium and plays an essential role in calcium-mediated regulation of inflammation in innate immune cells [12]. AMP-activated protein kinase (AMPK) is a crucial substrate of CaMKK $\beta$ which mainly functions as a cellular nutrient and energy sensor [15]. Recent studies reveal that AMPK is also an effective inhibitor for NF- $\kappa \mathrm{B}$ signaling and associated with inflammatory response in macrophages $[12,16]$. Sirtuin 1 (SIRT1) is a key downstream regulator of AMPK in metabolism. Recently, SIRT1 has been identified to be required for AMPK-mediated inflammation inhibition [17]. As a NAD ${ }^{+}$-dependent deacetylating enzyme, SIRT1 could be activated by elevated $\mathrm{NAD}^{+}$levels and then downregulates inflammation by directly deacetylating subunit of NF- $\kappa \mathrm{B}$ like p65 and Cel $[18,19]$. In addition, SIRT1 could also reciprocally activate AMPK or induce phosphorylation of PCG-1 $\alpha$, which thereafter inhibits the RelA/p65-mediated NF- $\kappa$ B signaling. Despite the well known effects of CaMKK $\beta$, AMPK, and SIRT1 on the modulation of inflammation, their concomitant involvement in mediating the regulation of calcium-dependent IL-12 production needs to be further studied.

In this study, we detected calcium influx across the plasma membrane triggered by LPS and observed its inhibitory effect on LPS induced IL-12 secretion in murine macrophages. We also evaluated the effects of CaMKK $\beta$-AMPK-SIRT1 signaling pathway on regulating NF- $\kappa \mathrm{B}$ activity and mediating calcium-dependent IL-12 inhibition.

\section{Materials and Methods}

2.1. Cell Culture. Murine peritoneal macrophages were obtained from peritoneal lavage in $\mathrm{BALB} / \mathrm{c}$ male mice as described previously [20]. Briefly, BALB/c mice were sacrificed and $1 \mathrm{~mL}$ high glucose DMEM (GIBCO, USA) was injected into the intraperitoneal cavity. The abdomen was gently massaged for $1 \mathrm{~min}$ and the injected medium was aspirated and cell pellets were washed twice with DMEM containing $10 \%$ fetal bovine serum (FBS). Then, peritoneal macrophages were plated in plates or dishes and cultured at $37^{\circ} \mathrm{C}$ in a humidified incubator supplemented with $5 \% \mathrm{CO}_{2}$. Murine macrophage-like cell line RAW 264.7 cells (ATCC, USA) were directly cultured in DMEM supplemented with $10 \%$ FBS and under similar environment with primary macrophages.

2.2. Cell Treatment. For calcium modulation, macrophages were treated in calcium-free DMEM (GIBCO) or DMEM with EGTA (Sigma, USA) for calcium deprivation. Cells were treated with calcium-free DMEM plus $2 \mathrm{mM} \mathrm{CaCl}_{2}$ (Sigma) for calcium recovery. Then, macrophages were also treated with SKF96365, 2-aminoethyl diphenylborinate (2APB) (Sigma), and $\mathrm{LaCl}_{3}$ (Aladdin, China) for inhibition of store-operated calcium entry (SOCE) or with ATP to increase calcium ATPase activity. Then, LPS O55: B5 (Sigma) was added and incubated for the indicated time periods before further measurement. For chemical modulation of CaMKK $\beta$, AMPK, SIRT1, NF- $\kappa$ B, and AP-1, macrophages were pretreated with STO-609, AICAR, Compound C, wedelolactone (Sigma), SR11302 (Tocris, UK), and SRT1720 and EX527 (Selleck, USA) for $1 \mathrm{~h}$ and then stimulated with LPS before further measurement.

2.3. Intracellular Calcium Detection. Time-dependent intracellular calcium detection was performed with a Varioskan ${ }^{\mathrm{TM}}$ Flash Multimode Reader (Thermo) using the fluorescent dye Fura-2AM (Sigma). Murine peritoneal macrophages were seeded in 96-well plates and preloaded with Fura-2AM for $60 \mathrm{~min}$. Loaded cells were treated with or without LPS $(1 \mu \mathrm{g} / \mathrm{mL})$. Online detection of Fura-2AM in live cells was performed every $10 \mathrm{~s}$ for $6 \mathrm{~min}$ via fluorescence excited at 340 and $380 \mathrm{~nm}$ and filtered at $510 \mathrm{~nm}$. Ratios of $340 / 380 \mathrm{~nm}$ were calculated and compared.

2.4. siRNA Transient Transfection. CaMKK $\beta$, AMPK $\alpha$, and SIRT1 were transiently knocked down by siRNA transfection following the manufacturer's instruction. Briefly, RAW 264.7 cells were cultured into $70 \%$ confluence. Negative control (NC) siRNA (Santa Cruz, USA) or siRNA for CaMKK $\beta$, AMPK $\alpha$ (Ruibo Biotech Ltd., China), and SIRT1 (Santa Cruz) were mixed with Lipofectamine 3000 reagent (Invitrogen, USA) and added to the medium. After $24 \mathrm{~h}$ of transfection, the culture medium was replaced and further treatment was performed.

2.5. Western Blot Analysis. RAW 264.7 cells were lysed with RIPA reagents (Pierce, Rockford, IL, USA) containing cocktail protease and phosphatase inhibitors (Roche, Switzerland). Plasma proteins were separated by SDS-PAGE and transferred onto PVDF membranes (Millipore, USA). Blots were blocked with $5 \%$ BSA for $1 \mathrm{~h}$ and incubated with primary antibodies (1:1000 dilutions) for CaMKK $\beta, \mathrm{pCaMKK} \beta$, AMPK $\alpha, \mathrm{pAMPK} \alpha$, SIRT1, I $\kappa \mathrm{B} \alpha$, IRF3, pIRF3, p38, p-p38, 
and tubulin (Cell Signaling, USA) at $4^{\circ} \mathrm{C}$ overnight. Then, the blots were further incubated with HRP-conjugated secondary IgG antibodies (1:2000 dilutions, Cell Signaling) at $37^{\circ} \mathrm{C}$ for $1 \mathrm{~h}$. Chemiluminescence images were developed with SuperSignal Sensitivity Substrate Kit (Pierce) with a ChemiDoc XRS imaging system (Bio-Rad, USA).

2.6. Real-Time PCR. Total RNA extracted from RAW 264.7 cells by a TRIzol reagent (Roche) was reversely transcribed into cDNA with a ReverTra Ace- $\alpha$-RNA easy Kit (Toyobo, Japan). The cDNA templates were mixed with SYBR Green PCR Master Mix (Toyobo) and PCR primers for IL-12 p35, IL-12 p40, TNF- $\alpha$, IL-6, CaMKK $\beta, \operatorname{AMPK} \alpha$, and $\beta$-actin (sequences are listed in Table S1, in Supplementary Material available online at http://dx.doi.org/10.1155/2016/6152713). Quantitative real-time PCR was performed with an iCycler Thermal Cycler (Bio-Rad).

2.7. Luciferase Reporter Gene Assay. RAW 264.7 cells $(1 \times$ $10^{5} / \mathrm{mL}$ ) were cultured in a 24 -well plate and transfected with plasmids pGL-luc2P/NF- $\kappa$ BRE (Promega, USA) and pAP1-luc and pIFN- $\beta$-luc (Genepharma, China) using XtremeGENE HP DNA Transfection Reagent (Roche) for $24 \mathrm{~h}$. Then, the plate was added with LPS for $6 \mathrm{~h}$ of incubation treatment. The luciferase activity was analyzed with the Firefly Luciferase Assay Kit (Beyotime) and detected in a multimode reader (Thermo). Relative luciferase light units were normalized to untreated cells.

2.8. ELISA Assay. Supernatants from cultured murine peritoneal macrophages or RAW 264.7 cells were directly collected after $24 \mathrm{~h}$ of LPS treatment or at a given time. The levels of IL-12 p40 and IL-12 p70 in the supernatant were measured with ELISA Kits (eBioscience, USA) as indicated by the manufacture's instruction.

2.9. $\mathrm{NAD}^{+} / \mathrm{NADH}$ Ratio Assay. RAW 264.7 cells $\left(2 \times 10^{5} / \mathrm{mL}\right)$ growing in 24-well plates were treated with LPS and LPS plus calcium-free DMEM and calcium-free DMEM with $\mathrm{CaCl}_{2}$ for $1 \mathrm{~h}$. Cells were washed twice with $\mathrm{PBS}$. NAD ${ }^{+} / \mathrm{NADH}$ levels in cell lyses were detected through a NADH Quantitative Detection Kit (Sigma). The procedures were performed as indicated by the manufacturer's instruction.

2.10. Statistical Analysis. Quantitative data were expressed as means \pm standard deviation (SD). Student's $t$-test was used for comparisons between two groups. One-way ANOVA plus post hoc Bonferroni correction was used for multiple comparisons. Difference with a $P$ value less than 0.05 and 0.01 was considered to be statistically significant.

\section{Results}

3.1. LPS Induces Secretion of TNF- $\alpha$, IL-6, and IL-10 in Large Amounts but Stimulates a Much Lower Level of IL-12 Production in Murine Macrophages. To compare supernatant levels of TNF- $\alpha$, IL-6, IL-10, and IL-12 (p40 and p70), primary murine peritoneal macrophages were treated with $100 \mathrm{ng} / \mathrm{mL}$
LPS. Results showed LPS induced dramatic increase of TNF- $\alpha$, IL-6, and IL-10 secretion but triggered relatively lower concentration of IL-12 p40 and IL-12 p70 production (Figure 1(a)). Moreover, LPS (10, 100, and $1000 \mathrm{ng} / \mathrm{mL}$ ) induced robust increase of TNF- $\alpha$ secretion in a significantly dose-dependent manner, but IL-12 secretion only increased slightly with elevated LPS concentration (Figure 1(b)). In the time course detection, the production of TNF- $\alpha$ increased rapidly $6 \mathrm{~h}$ after LPS stimulation. IL-12 p40 and IL-12 p70 were also elevated time dependently. However, their increases were much weaker (Figure 1(c)). Consistent trend was also detected in LPS-treated RAW 264.7 cells (Figure S1).

3.2. The Absence of Extracellular Calcium Selectively Upregulated IL-12 Expression in LPS-Treated Macrophages. The influence of extracellular calcium on cytokines production was investigated by treating murine peritoneal macrophages in DMEM with or without calcium (calcium-free). The results showed there was no difference in the expression of TNF- $\alpha$ or IL- 6 regardless of whether the medium contained calcium or not (Figures 2(a) and 2(b)). However, IL-12 p40 and IL-12 p70 were significantly upregulated without calcium. Similar changes of TNF- $\alpha$ and IL-12 p40 were detected in RAW 264.7 cells (Figure S2). Moreover, LPS induced production of IL-12 p40 was enhanced by cotreatment with IFN- $\gamma$. Deprivation of calcium further increased the IL-12 p40 production (Figure S3). The specificity of LPS induced IL-12 alteration due to calcium modulation was also identified by PMB cotreatment in RAW 264.7 cells. This specific LPS neutralizer effectively suppressed the elevated IL-12 p40 and IL- 6 regardless of whether calcium is present or absent (Figure S4). To fully elucidate the influence of extracellular calcium on IL-12 production, murine peritoneal macrophages were treated in calcium-free DMEM with or without $\mathrm{CaCl}_{2}$ supplementation. Cells were also treated with EGTA, a calcium chelator. Calcium-free DMEM and EGTA consistently increased the secretion of IL-12 p40 and IL-12 p70 proteins. Accordingly, mRNA expressions of IL-12 p35 and IL-12 p40 were also significantly upregulated (Figures 2(c) and 2(d)). The elevated expression of IL-12 was terminated when $\mathrm{CaCl}_{2}$ was resupplied, suggesting that the inhibitory effect was calciumdependent.

3.3. Depletion of Extracellular Calcium Dampens the Influx of Calcium and Thereby Inhibits IL-12 Expression in LPSStimulated Murine Macrophages. Next, we detected the calcium influx in macrophages by LPS treatment. A transient elevation of intracellular calcium levels (indicated by Fura2AM) was detected after LPS stimulation. The peak time came at 1-2 min after LPS treatment. Moreover, LPS induced increase of intracellular calcium levels was impaired when extracellular free calcium was removed (Figure 3(a)). These data suggest that transmembrane influx was induced by LPS which increased intracellular calcium levels. Besides direct extracellular calcium modulation, we further pretreated macrophages with SKF96365 and ATP, which either inhibited or activated the influx of calcium. In our study, SKF96365 treatment attenuated the influx of calcium and then resulted 


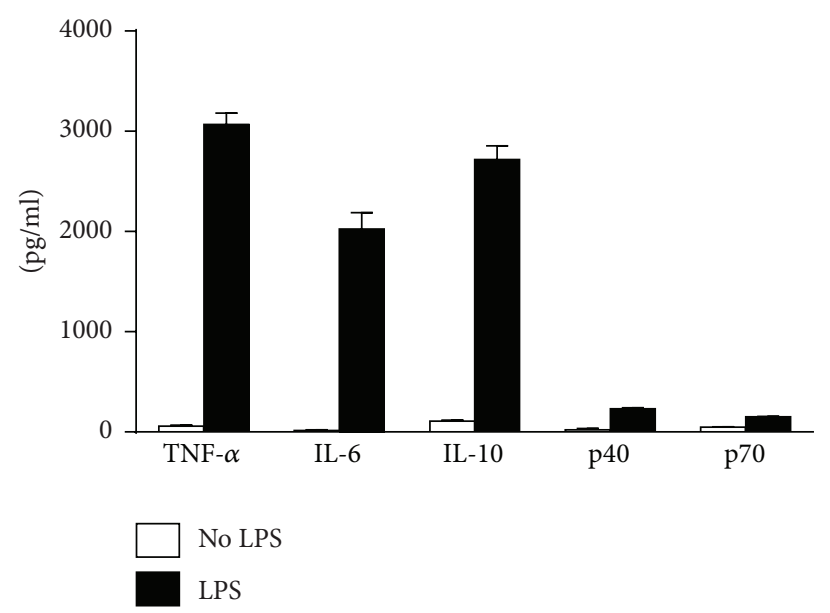

(a)
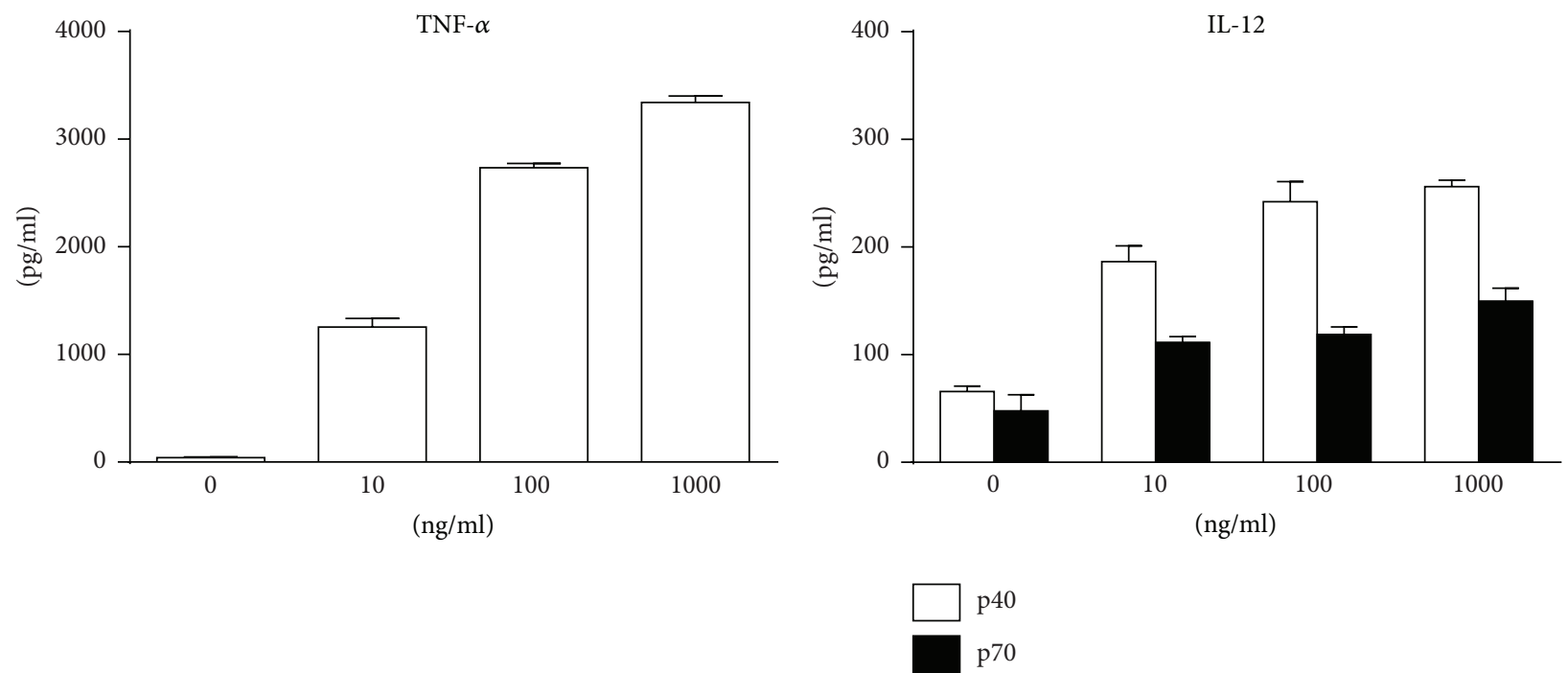

(b)
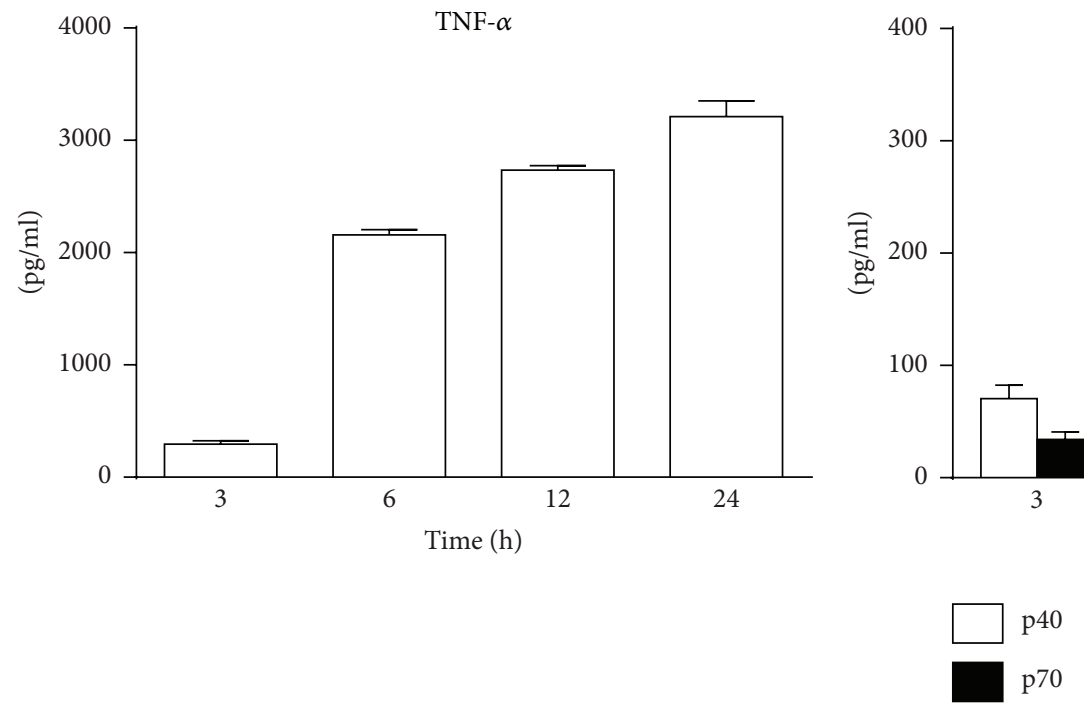

(c)

FIGURE 1: LPS induces the low levels of IL-12 in murine peritoneal macrophages. (a) Murine peritoneal macrophages were treated with or without $100 \mathrm{ng} / \mathrm{mL}$ LPS for $24 \mathrm{~h}$. Supernatant levels of TNF- $\alpha$, IL-6, IL-10, IL-12 p40, and IL-12 p70 were detected by ELISA. (b, c) Peritoneal macrophages were treated with LPS $(0,10,100$, and $1000 \mathrm{ng} / \mathrm{mL}$ ) for $24 \mathrm{~h}$ (b) or with $100 \mathrm{ng} / \mathrm{mL}$ LPS for 3, 6, 12, and 24 h (c). Supernatant levels of TNF- $\alpha$ and IL-12 p40/p70 were detected by ELISA $(n=3)$. 

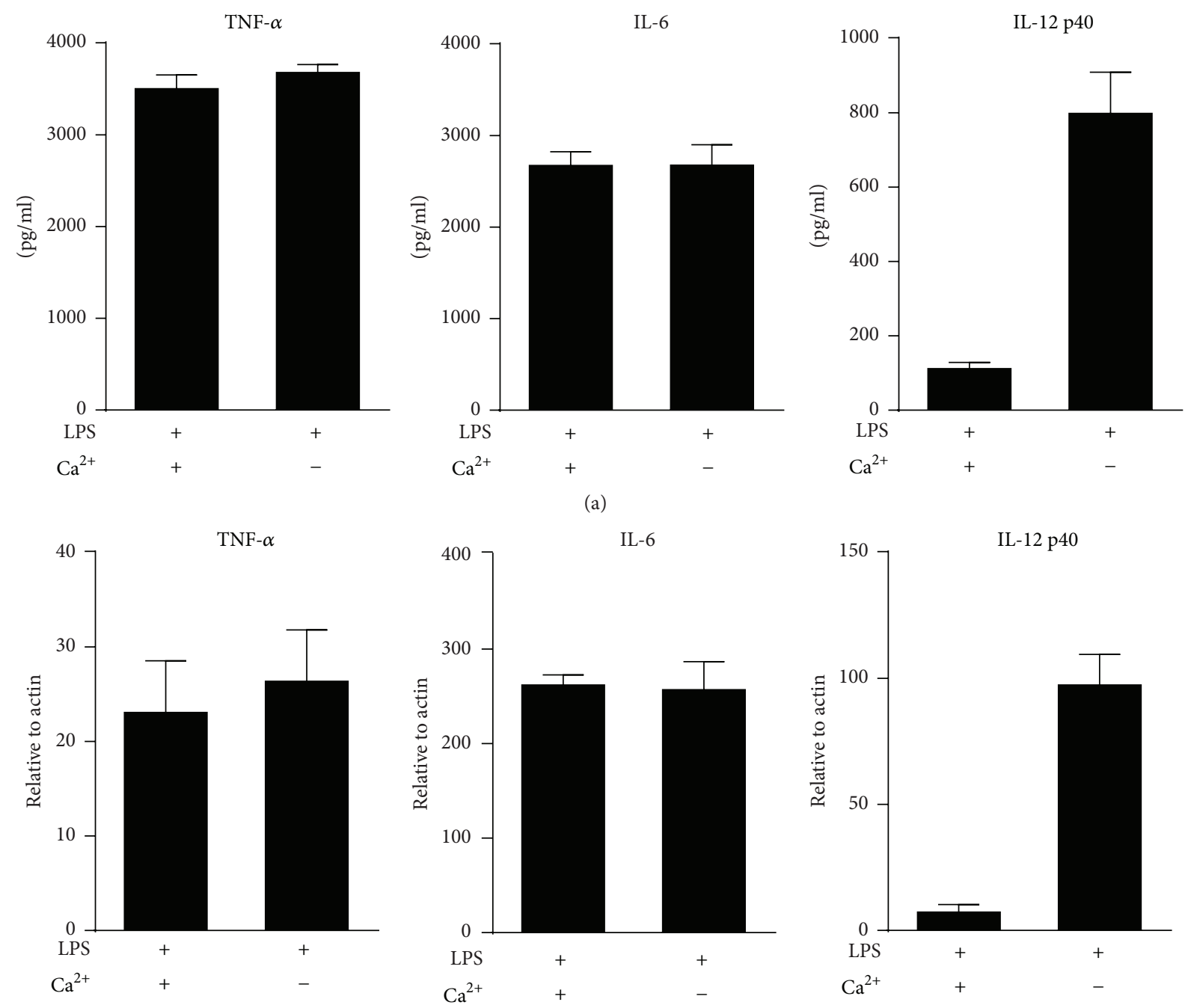

(b)

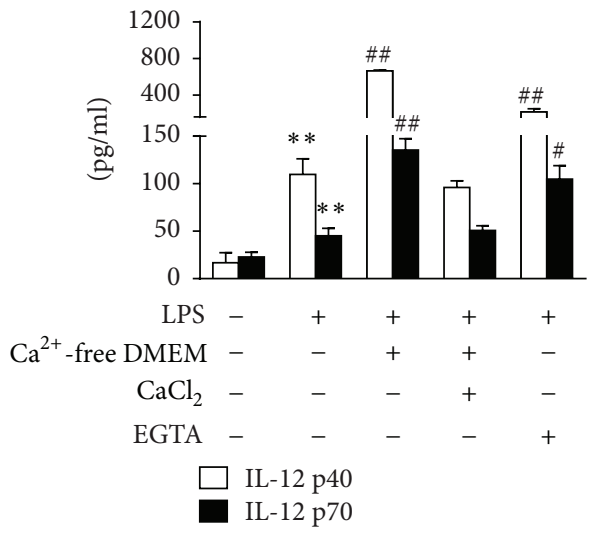

(c)

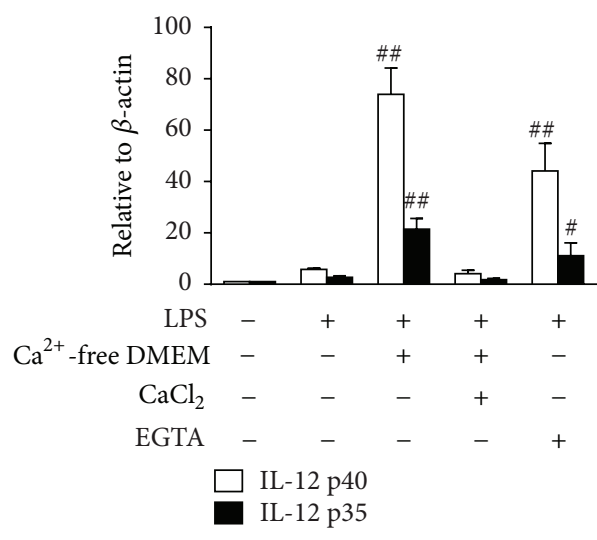

(d)

FIGURE 2: Depletion of extracellular calcium selectively upregulates IL-12 expression in LPS-treated murine macrophages. (a, b) Murine peritoneal macrophages cultured in DMEM with or without calcium for $1 \mathrm{~h}$ and then stimulated with LPS (100 ng/mL). Supernatants were collected after $24 \mathrm{~h}$ and the protein levels of TNF- $\alpha$, IL- 6 , and IL-12 p40 were detected by ELISA ((a) $n=3)$. Total RNA was extracted after $12 \mathrm{~h}$ and mRNA expressions of TNF- $\alpha$, IL- 6 , and IL-12 p40 were detected by real-time PCR ((b) $n=3) .{ }^{* *} P<0.01$ versus LPS. (c, d) Murine peritoneal macrophages were incubated with LPS $(100 \mathrm{ng} / \mathrm{mL})$ in normal DMEM, calcium-free DMEM, calcium-free DMEM with $2 \mathrm{mM}$ $\mathrm{CaCl}_{2}$, or normal DMEM with $5 \mathrm{mM}$ EGTA. The mRNA expressions of IL-12 p40 and IL-12 p35 were detected via real-time PCR (c). The supernatant levels of IL-12 p40 and IL-12 p35 were detected via ELISA (d). ${ }^{* *} P<0.01$ versus medium; ${ }^{*} P<0.05$ and ${ }^{\# \#} P<0.01$ versus LPS $(n=3)$. 


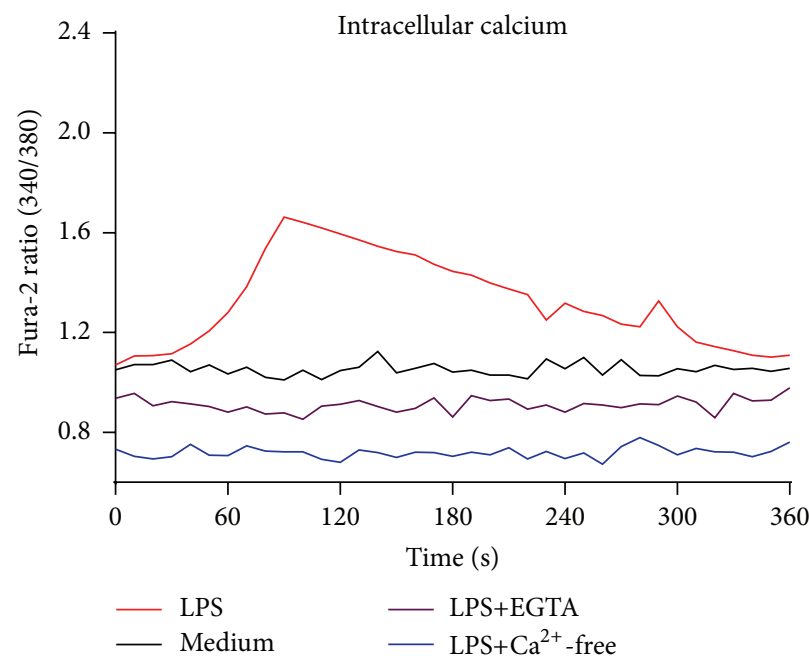

(a)

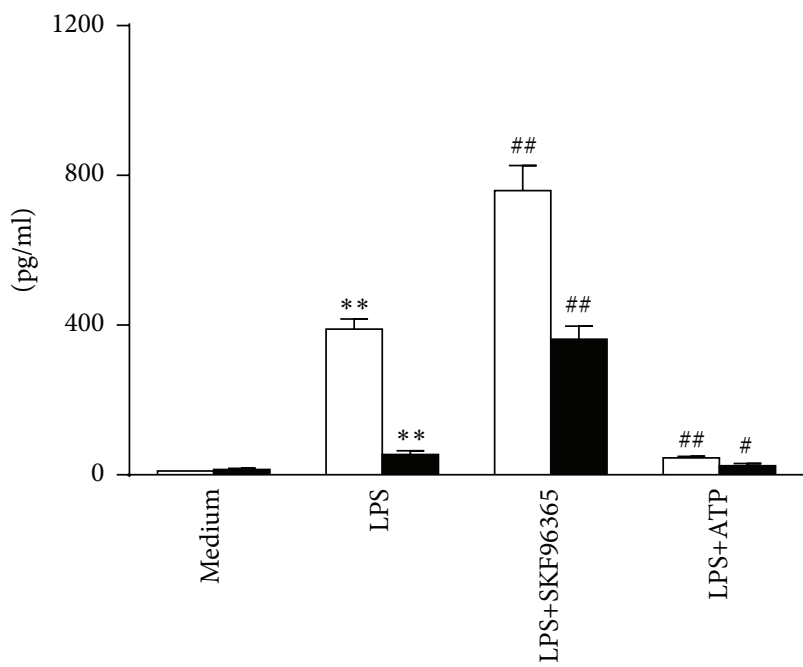

IL-12 p40

IL-12 p70

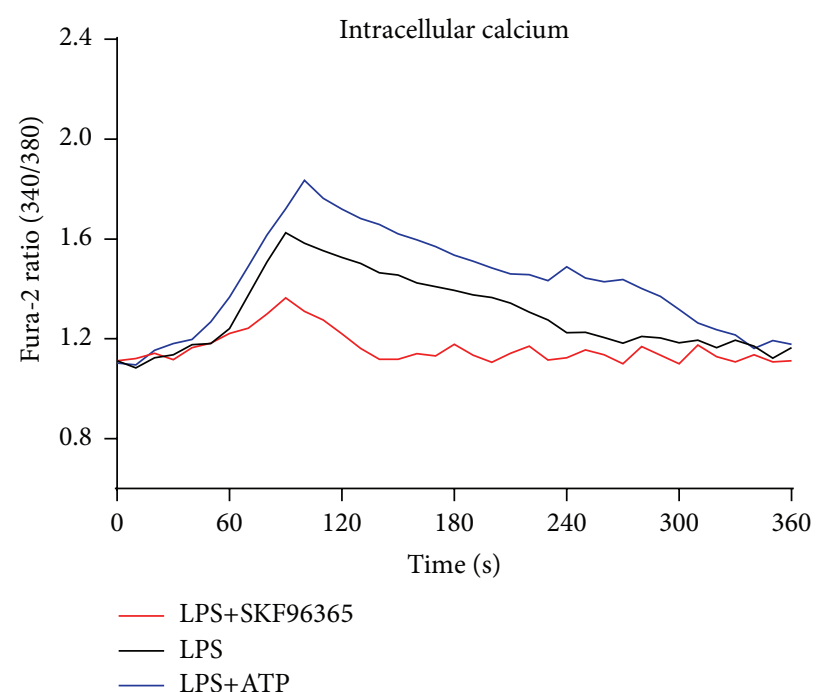

(b)

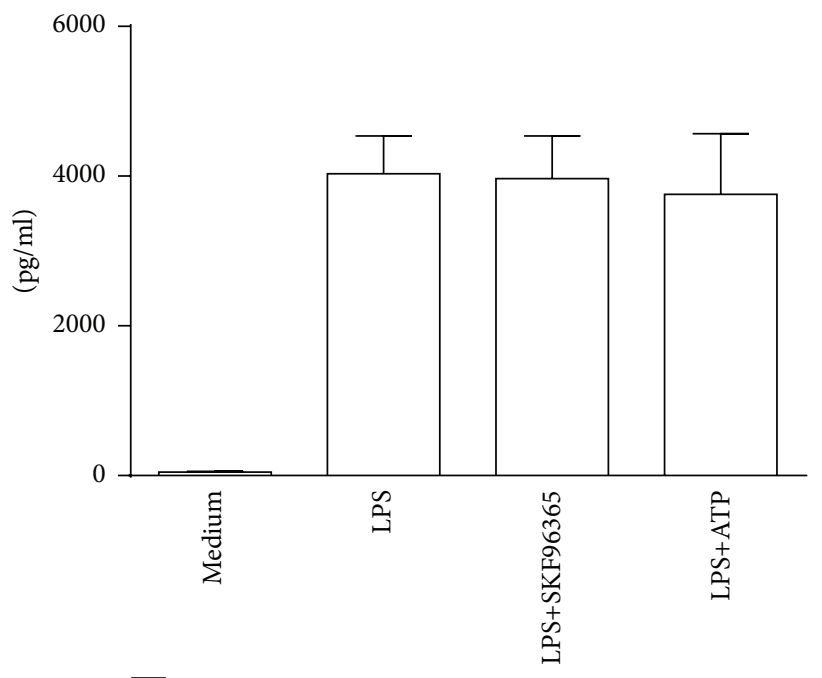

TNF- $\alpha$

(c)

Figure 3: LPS induced extracellular calcium influx and modulation of calcium influx affects IL-12 production in murine peritoneal macrophages. ( $\mathrm{a}, \mathrm{b})$ Macrophages were incubated with LPS $(1 \mu \mathrm{g} / \mathrm{mL})$ or LPS plus calcium-free DMEM, $5 \mathrm{mM}$ EGTA, $5 \mu$ M SKF96365, or $100 \mu \mathrm{M}$ ATP for $6 \mathrm{~min}$. The fluorescence ratio $(340 \mathrm{~nm} / 380 \mathrm{~nm})$ of Fura-2AM was captured by using the plate reader assay every $10 \mathrm{~s}(n=4)$. (c) Macrophages were treated with $5 \mu \mathrm{M}$ SKF96365 (SKF) or $100 \mu \mathrm{M}$ ATP for $1 \mathrm{~h}$ and then stimulated with $100 \mathrm{ng} / \mathrm{mL}$ LPS. Supernatants were collected $24 \mathrm{~h}$ later and IL-12 p40 and IL-12 p70 levels were detected by ELISA. ${ }^{* *} P<0.01$ versus medium; ${ }^{\#} P<0.05$ versus LPS; ${ }^{\# \#} P<0.01$ versus LPS $(n=3)$.

in the elevation of IL-12 p40 and IL-12 p70 production. In contrast, ATP treatment enhanced calcium influx and further decreased IL-12 secretion upon LPS stimulation. However, TNF- $\alpha$ release was not affected by SKF96365 or ATP (Figures 3(b) and 3(c)). We further detected the IL12 modulating effects of other SOCE inhibitors (2-APB and $\mathrm{LaCl}_{3}$ ) and observed similar effects with SKF96365 (Figure S5). These results indicated that LPS induced intracellular calcium elevation by triggering extracellular calcium entry, which is also required for the negative regulation of IL-12 production in macrophages.
3.4. The Activation of CaMKK $\beta$ Mediates Calcium-Dependent Inhibition of IL-12 Production in LPS-Activated Macrophages. To identify the downstream signaling effectors in calciummediated IL-12 inhibition, we detected CaMKK $\beta$ activation in RAW 264.7 cells. In our study, LPS induced phosphorylation of $\mathrm{CaMKK} \beta$ was suppressed in medium without calcium or EGTA, both of which inhibited extracellular calcium influx. Restoration of CaMKK $\beta$ phosphorylation was observed when extracellular calcium was resupplied (Figure 4(a)). To investigate whether activation of CaMKK $\beta$ leads to IL-12 inhibition, we used chemical inhibitor 


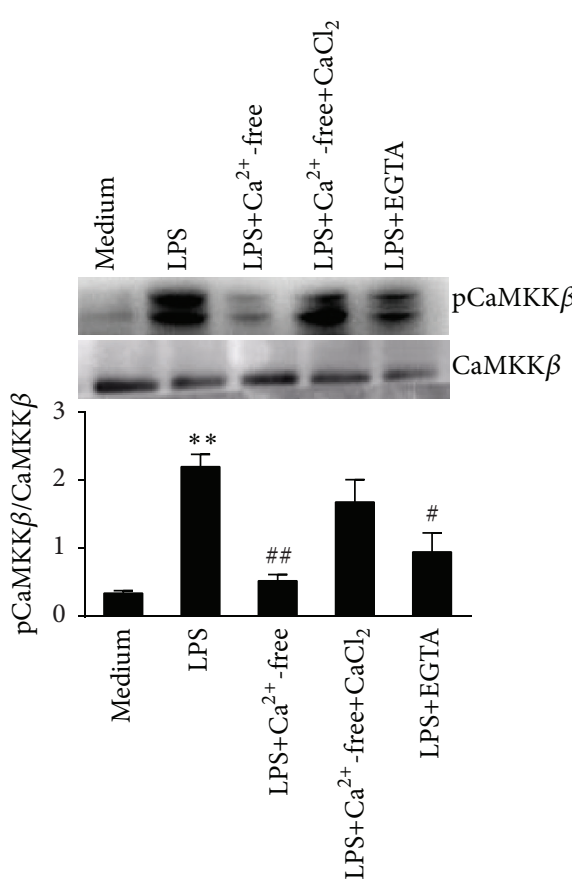

(a)

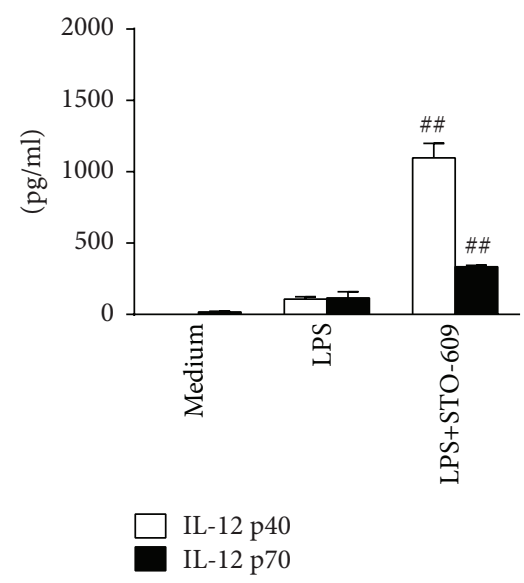

(b)

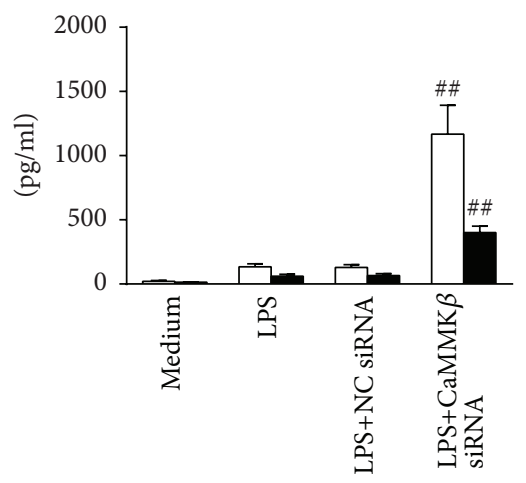

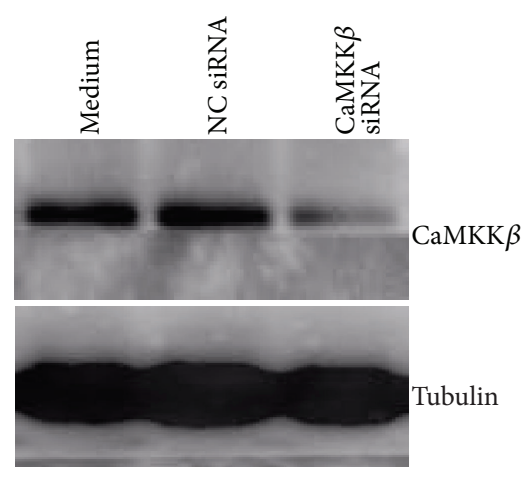

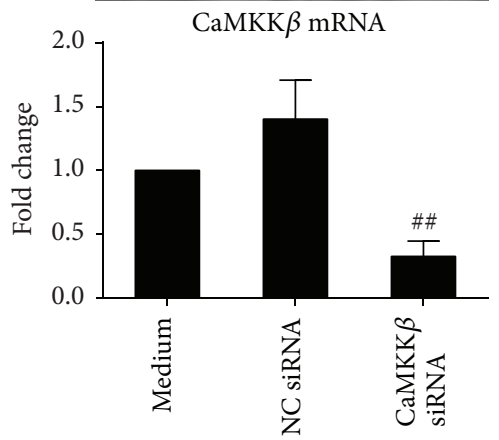

(c)

IL-12 p40
IL-12 p70

(d)

FIgURE 4: Activation of CaMKK $\beta$ mediates the inhibition of calcium-dependent IL-2 production in RAW 264.7 cells. (a) Cells were treated with LPS in normal DMEM, calcium-free DMEM, calcium-free DMEM with $2 \mathrm{mM} \mathrm{CaCl}_{2}$, or DMEM with $5 \mathrm{mM}$ EGTA for 30 min. Protein levels of $\mathrm{CaMKK} \beta$ and $\mathrm{pCaMKK} \beta$ were detected by western blot. Comparison was made for pCaMKK $\beta /$ CaMKK $\beta$. ${ }^{* *} P<0.01$ versus medium; ${ }^{\#} P<0.05$ and ${ }^{\# \#} P<0.01$ versus LPS $(n=3)$. (b) Cells were treated with LPS or LPS plus $1 \mu \mathrm{M}$ STO-609 for $24 \mathrm{~h}$. Supernatants IL-12 p40 and IL-12 p70 were detected. ${ }^{\# \#} P<0.01$ versus LPS for each cytokine $(n=3)$. (c) Cells were treated with negative control siRNA (NC siRNA) or CaMKK $\beta$ siRNA for $24 \mathrm{~h}$. mRNA and protein levels of CaMKK $\beta$ were detected by real-time PCR or western blot. ${ }^{\# \#} P<0.01$ versus NC siRNA $(n=3)$ for mRNA. (d) siRNA-treated cells were further stimulated by $100 \mathrm{ng} / \mathrm{mL}$ LPS for 24 h. Supernatants IL-12 p40 and IL-12 p70 were detected. ${ }^{\# \#} P<0.01$ versus LPS for each cytokine $(n=3)$.

STO-609 to suppress CaMKK $\beta$ activation and found that STO-609 increased IL-12 production but did not affect the release of TNF- $\alpha$ induced by LPS (Figures 4(b) and S6). We further transfected specific siRNA to directly downregulate CaMKK $\beta$ expression (Figure 4(c)). IL-12 production significantly increased in CaMKK $\beta$ siRNA-treated groups $(P<$ 0.01 versus LPS, Figure $4(\mathrm{~d})$ ). Such results demonstrate that the activation of CaMKK $\beta$ is required for calcium associated IL-12 suppression in LPS-treated macrophages.
3.5. AMPK Is Activated Downstream of CaMKK $\beta$ and Required for the Suppression of LPS Induced IL-12 Production in Macrophages. Next, we investigated whether AMPK was activated downstream of CaMKK $\beta$ to mediate IL-12 inhibition. In our study, suppressed AMPK $\alpha$ phosphorylation (Thr 172) was detected in RAW 264.7 cells stimulated by LPS in calcium-free medium. AMPK phosphorylation was restored by supplementing $\mathrm{CaCl}_{2}$ recovery in the medium (Figure 5(a)). Moreover, EGTA and STO-609 also inhibited 


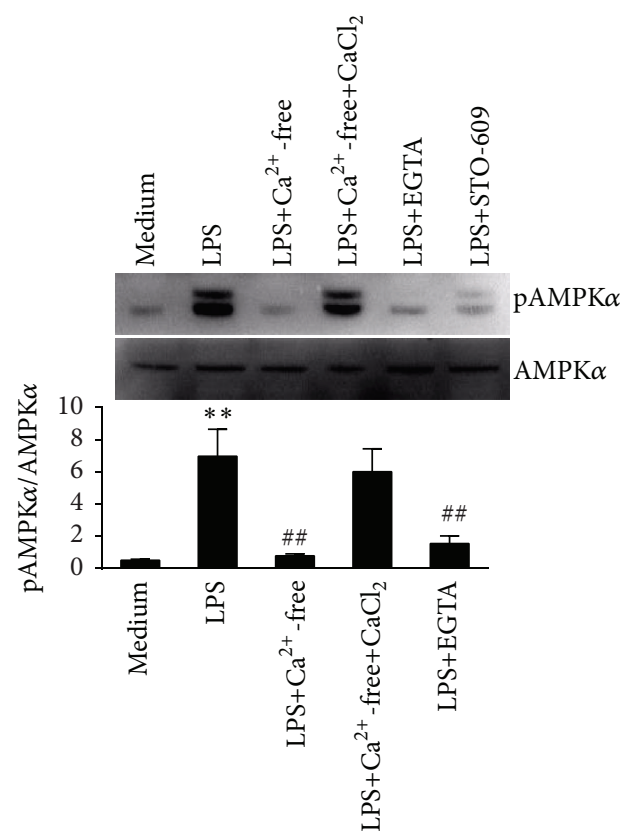

(a)
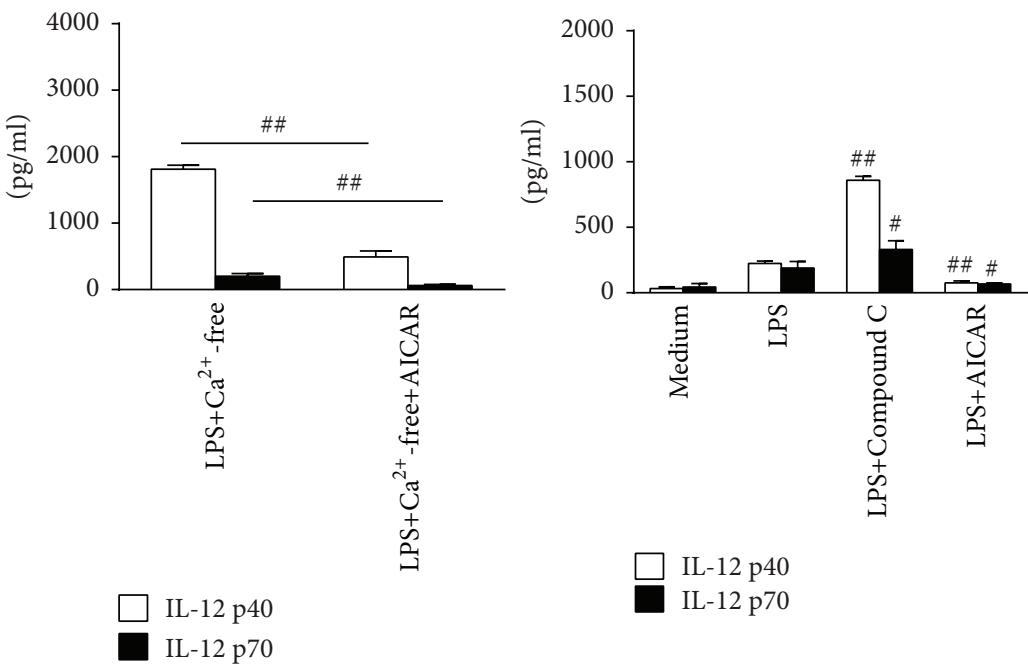

(b)

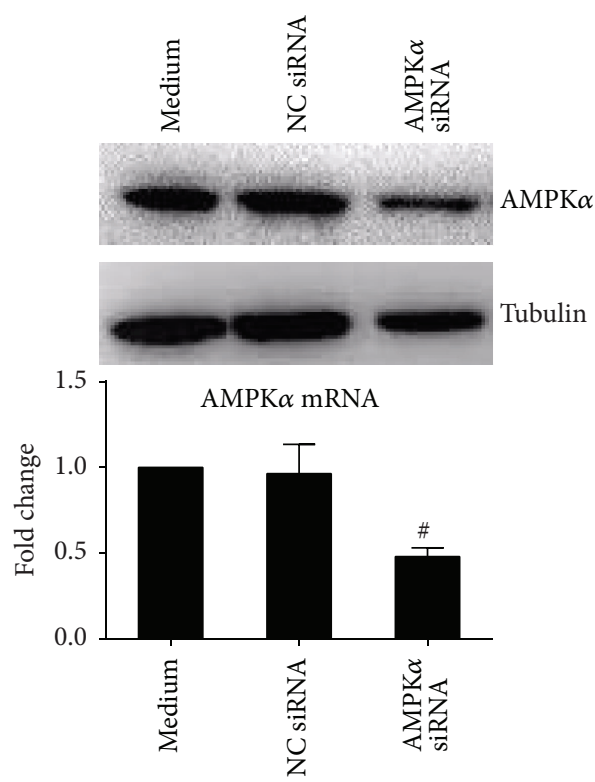

(c)

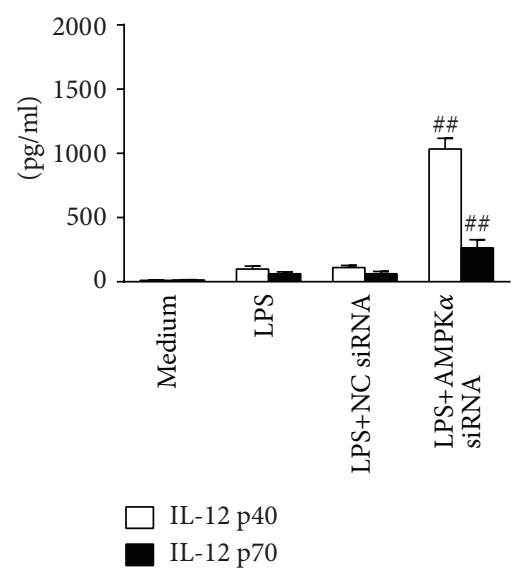

(d)

FIgURE 5: AMPK is activated downstream of CaMKK $\beta$ for the negative control of the LPS induced IL-12 production in RAW 264.7 cells. (a)

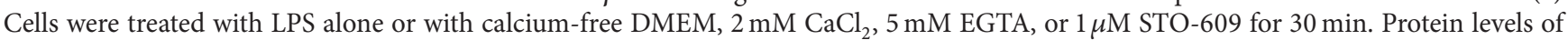
AMPK $\alpha$ and pAMPK $\alpha$ were detected by western blot. ${ }^{* *} P<0.01$ versus medium; ${ }^{\#} P<0.05$ or ${ }^{\# \#} P<0.01$ versus LPS ( $n=3$ ). (b) Cells were treated with LPS or LPS plus $1 \mathrm{mM}$ AICAR in calcium-free DMEM (left) or with LPS, LPS plus $5 \mu \mathrm{M}$ Compound C, and LPS plus $1 \mathrm{mM}$ AICAR in normal DMEM (right). Supernatants IL-12 p40 and IL-12 p70 were detected by ELISA. ${ }^{\#} P<0.05$ and ${ }^{\# \#} P<0.01$ versus LPS $(n=3)$. (c) Cells were treated with NC siRNA or AMPK $\alpha$ siRNA for $24 \mathrm{~h}$. mRNA and protein levels of AMPK $\alpha$ were detected by real-time PCR or western blot. ${ }^{\#} P<0.05$ versus NC siRNA $(n=3)$ for mRNA. (d) siRNA-treated cells were further stimulated by $100 \mathrm{ng} / \mathrm{mL}$ LPS for 24 h. Supernatants IL-12 p40 and IL-12 p70 (24 h) were detected. ${ }^{\#} P<0.05$ and ${ }^{\# \#} P<0.01$ versus LPS for each cytokine $(n=3)$.

AMPK $\alpha$ phosphorylation. We further treated RAW 264.7 cells with AICAR (AMPK activator) or Compound C (AMPK inhibitor) before LPS stimulation. Whereas Compound C caused significant increase of IL-12 secretion, AICAR further decreased the production of IL-12 (Figure 5(b)).
Then, addition of AICAR in calcium-free medium before LPS stimulation resulted in suppressed production of IL12 p40 and IL-12 p70 $\left(P<0.01\right.$ versus $\mathrm{LPS}+\mathrm{Ca}^{2+}$ free). We also transfected AMPK $\alpha$ siRNA in RAW 264.7 cells to directly decrease AMPK $\alpha$ expression (Figure 5(c)). 
Accordingly, the release of IL-12 p40 and IL-12 70 induced by LPS increased with AMPK $\alpha$ downregulation $(P<0.01$ versus LPS, Figure 5(d)). These data collectively demonstrate that AMPK is activated downstream of CaMKK $\beta$ and required for calcium-induced inhibition of IL-12 production in LPStreated murine macrophages.

3.6. SIRT1 Is a Key Mediator Upregulated by AMPK Activation and Responsible for the Expression of IL-12 Expression. SIRT1 is a substrate of AMPK in the regulation of inflammation, so we investigated whether SIRT1 was also required in mediating IL-12 inhibition. As SIRT1 activation is $\mathrm{NAD}^{+}$-dependent, we first detected the $\mathrm{NAD}^{+} / \mathrm{NADH}$ ratio in the presence or absence of extracellular calcium. In our study, LPS treatment alone induced elevated $\mathrm{NAD}^{+}$levels, which was dampened by using calcium-free medium and restored by addition of $\mathrm{CaCl}_{2}$ (Figure 6(a)). Then, expressions of SIRT1 were upregulated by both LPS and AMPK activator AICAR in RAW 264.7 cells (Figure 6(b)). In accordance with $\mathrm{NAD}^{+}$detection, LPS treatment in medium without calcium caused suppressed SIRT1 expression. Furthermore, the SIRT1 inhibitor, EX527, markedly increased the production of IL-12 p40 and IL-12 p70 while its agonist, SRT1720, suppressed the IL-12 secretion induced by LPS (Figure 6(c)). When SRT1720 was added in calcium-free medium before LPS stimulation, the enhanced IL-12 production was inhibited (Figure 6(d)). In addition, knockdown of SIRT1 by siRNA also markedly enhanced IL-12 production upon LPS stimulation in macrophages (Figure 6(e)). These data verify that SIRT1 may be essentially responsible for calcium-mediated IL-12 suppression.

3.7. SIRT1 Selectively Attenuates NF- $\kappa B$ Activation Which Thereby Suppresses IL-12 Expression in LPS-Activated Macrophages. As calcium deprivation affects activation of CaMKK $\beta$, AMPK, and SIRT1, we next used combined modulator of these kinases to identify their connection in mediating IL-12 regulation. Our results demonstrated that AMPK and SIRT1 activation impaired the increased IL-12 production due to CaMKK $\beta$ inhibition. Moreover, SIRT1 activation suppressed IL-12 released due to AMPK inhibition while SIRT1 inhibition partly restored IL-12 production suppressed by AMPK activation (Figure 7(a)).

SIRT1 suppresses inflammation driven by interfering with NF- $\kappa \mathrm{B}$-activation, so we next detected whether NF- $\kappa \mathrm{B}$ pathway was specifically targeted by calcium and SIRT1 to downregulate IL-12 expression. In our study, the transcriptional activity of NF- $\kappa \mathrm{B}$ induced by LPS was enhanced in calciumfree medium. Cotreatment with $\mathrm{CaCl}_{2}$, AICAR, or SRT1720 in calcium-free medium, which restored calcium influx or activated AMPK and SIRT1, suppressed the elevated NF- $\kappa \mathrm{B}$ activity (Figure 7(a)). However, the absence of extracellular calcium did not affect the transcriptional activity of IRF3 and c-Jun (a subunit of AP-1) induced by LPS (Figure 7(b)). Moreover, a significant decrease of IL-12 production was detected by the cotreatment of NF- $\kappa \mathrm{B}$ inhibitor wedelolactone with LPS. However, AP-1 inhibitor, SR11302, did not alter IL-12 levels. In a parallel study, both wedelolactone and SR11302 significantly attenuated IL-6 production in RAW 264.7 cells
(Figure 7(c)). Furthermore, we observed similar modulation on IL-12 p40 and IL- 6 by SR11302 and wedelolactone in the absence of calcium (Figure $7(\mathrm{~d})$ ). These results suggest that LPS induced calcium influx mediates IL-12 inhibition by ultimate suppression of the NF- $\kappa \mathrm{B}$ signaling pathway.

\section{Discussion}

IL-12 is essentially involved in host immune response and increased evidences have demonstrated the critical effects of IL-12 deficiency that result in immunosuppression and increased susceptibility to infection $[1,21,22]$. However, the precise regulatory mechanisms of IL-12 expression remain unclear and thereby are worthy of investigation. In the present study, we report the finding of a calcium-dependent mechanism that may possibly explain the insufficient IL12 expression in LPS-stimulated murine macrophages. This newly identified mechanism is verified to be based on observations that LPS stimulation induces extracellular calcium influx which activates CaMKK $\beta$, AMPK, and SIRT1, dampens NF- $\kappa \mathrm{B}$ activation, and eventually downregulated IL-12 production.

Macrophages are primary sources for the production of inflammatory cytokines when activated by pathogenic molecules such as LPS. However, levels of these cytokines can vary greatly due to the complicated and diversified regulatory mechanisms [23]. In our study, LPS-triggered production of IL-12 p40 and IL-12 p70 was significantly lower than those of TNF- $\alpha$, IL- 6 , and IL-10 secreted in the same cell population of either primary or cell line of murine macrophages. However, the underlying mechanism was unclear. Previously, it was found that LPS could activate the calcium-dependent signaling and regulate cytokine production in innate and adaptive immune cells [24-26]. Additional studies provided more specific data that calcium might influence the production of IL$12[12,13]$. In our study, the removal of extracellular calcium directly caused a selective suppression of IL-12 production upon LPS stimulation. Therefore, these data suggest calcium may act as an essential negative regulator for the production of IL-12.

Extracellular calcium flux in response to microbial stimuli has been demonstrated in both mouse macrophages and mouse DCs [27, 28]. More specifically, calcium influx mainly contributes to the increase of intracellular calcium in LPS-treated macrophages [11]. In our study, we consistently observed extracellular calcium influx induced by LPS treatment in murine peritoneal macrophages and also in RAW 264.7 cells (data not shown). Such a transmembrane transportation of calcium was then demonstrated to be associated with IL-12 regulation, as enhanced IL-12 production was detected in macrophages cultured in medium without calcium or with lack of free calcium (EGTA). It has been found that calcium influx is regulated by clathrin/dynamindependent endocytosis, calcium channel regulators, and calcium ATPases. For example, the dynamin inhibitor dynasore could block TRPV5/V6 activity and inhibit calcium influx in Jurkat cells [29]. We found previously that inhibition of endocytic pathway significantly suppressed LPS induced production of IL-12 p40 [14]. SOCE is a primary process 


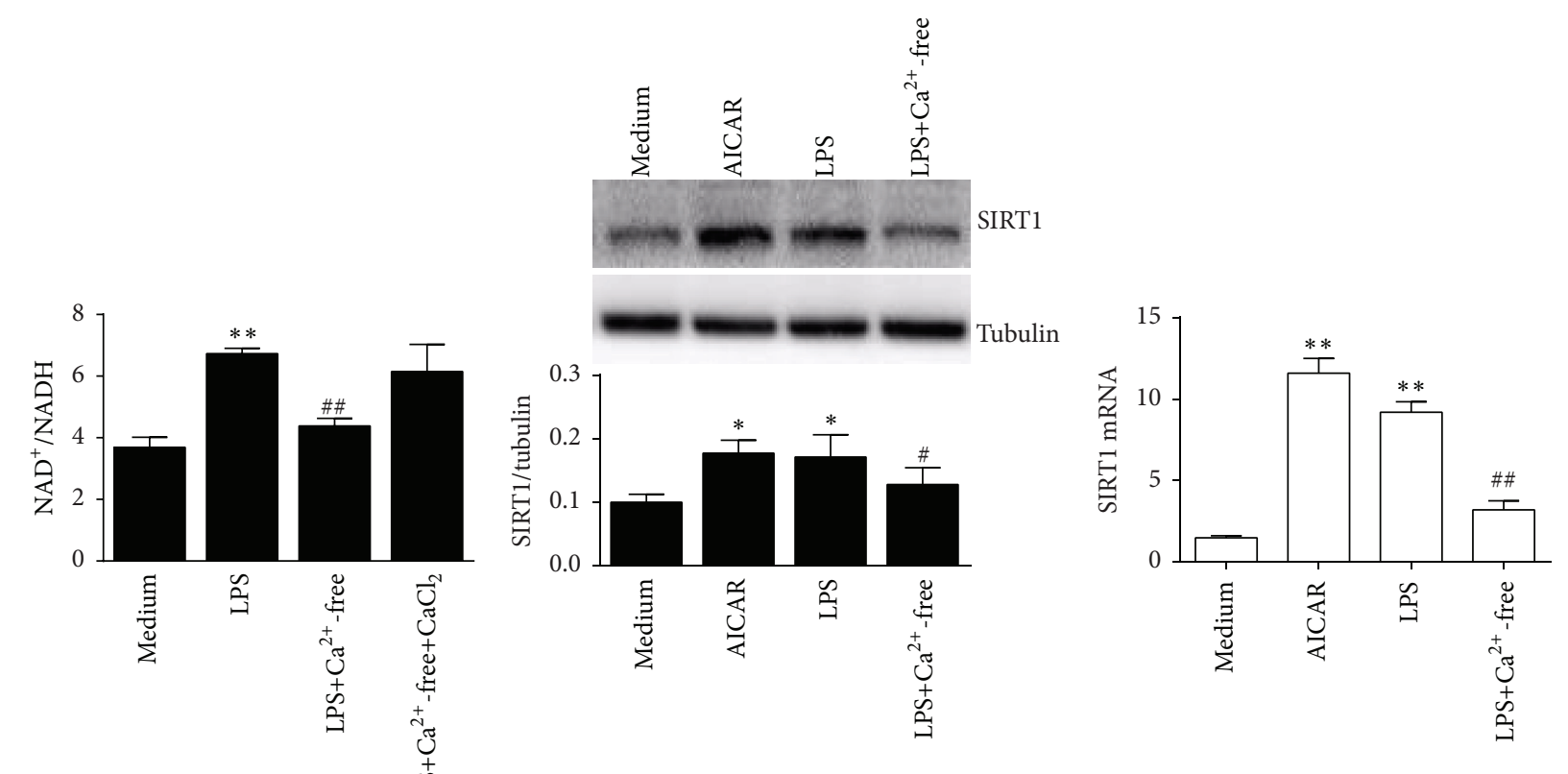

(a)

(b)

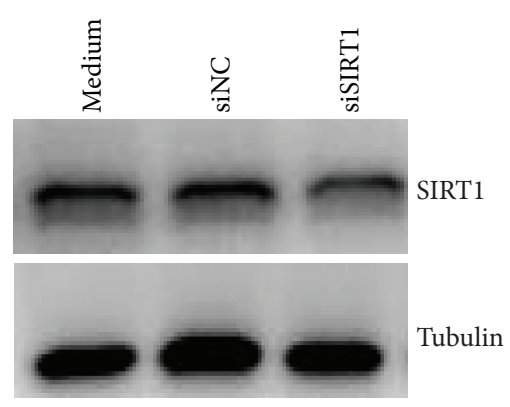

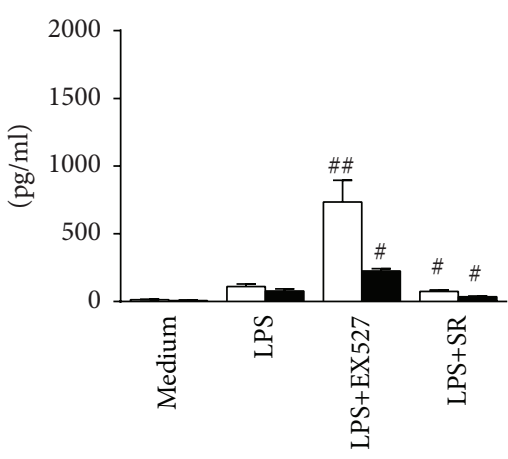

IL-12 p 40

IL-12 p70

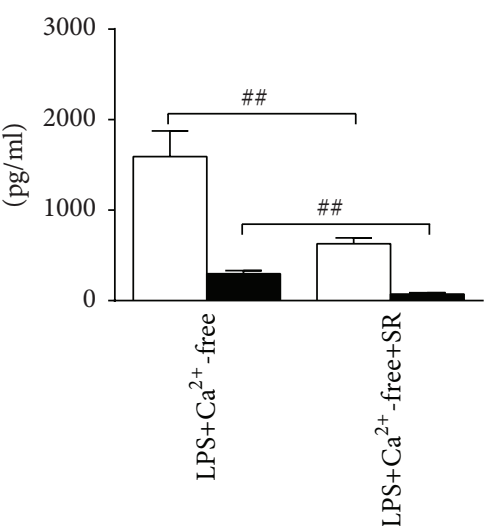

$\square$ IL-12 p40

IL-12 p70

(d)

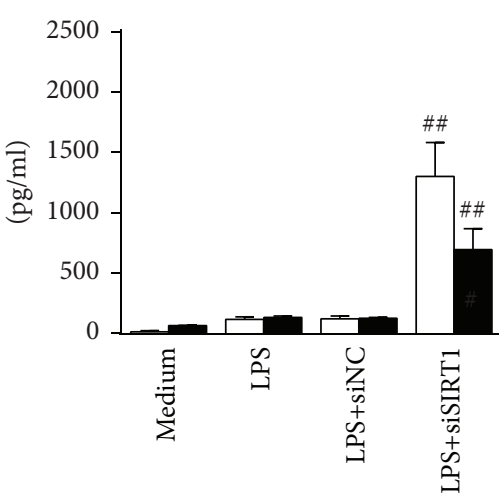

IL-12 p40

IL-12 p70

(c)

(e)

FIGURE 6: SIRT1 is upregulated by AMPK activation to suppress IL-12 expression in the LPS-activated RAW 264.7 cells. (a) Cells were treated with $100 \mathrm{ng} / \mathrm{mL}$ LPS in normal DMEM or in DMEM without calcium or supplemented with $2 \mathrm{mM} \mathrm{CaCl}_{2}$ for $1 \mathrm{~h}$. NAD $/ \mathrm{NADH}^{+}$levels were detected $(n=3)$. (b) Cells were treated with $1 \mathrm{mM}$ AICAR, $100 \mathrm{ng} / \mathrm{mL}$ LPS, or LPS plus calcium-free DMEM for $2 \mathrm{~h}$. Protein and mRNA levels of SIRT1 were detected by western blot or real-time PCR. ${ }^{*} P<0.05$ and ${ }^{* *} P<0.01$ versus medium; ${ }^{\#} P<0.05$ or ${ }^{\# \#} P<0.01$ versus LPS $(n=3)$. (c, d) Cells were treated with LPS alone or with $2 \mu \mathrm{M}$ EXT527 or $2 \mu \mathrm{M}$ SRT1720 (c). Cells were also treated with LPS, calcium-free LPS, or calcium-free LPS with SRT1720 (d). Supernatants IL-12 p40 and IL-12 p70 (24 h) were detected by ELISA. ${ }^{\#} P<0.05$ and ${ }^{\# \#} P<0.01$ versus LPS for each cytokine $(n=3)$. (e) Cells were treated with NC siRNA or SIRT1 siRNA for $24 \mathrm{~h}$. Protein levels of SIRT1 were detected by western blot (upper). The siRNA-treated cells were further stimulated by $100 \mathrm{ng} / \mathrm{mL}$ LPS for $24 \mathrm{~h}$ (lower). Supernatants IL-12 p40 and IL-12 p70 were detected. ${ }^{\#} P<0.05$ and ${ }^{\# \#} P<0.01$ versus LPS for each cytokine $(n=3)$. 


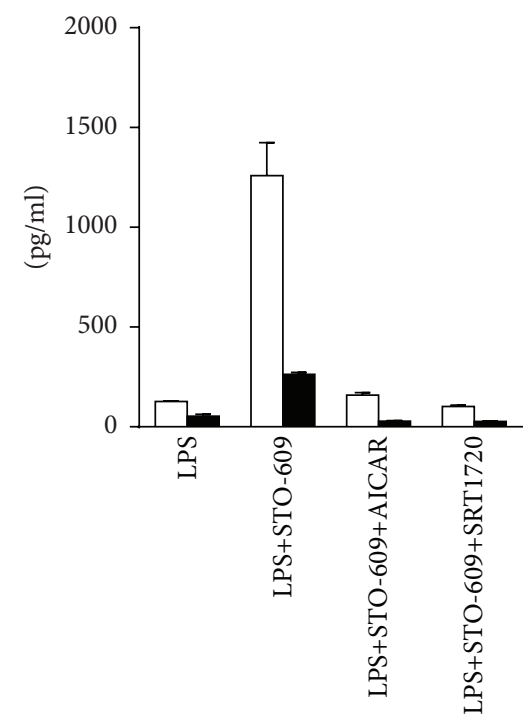

IL-12 p 40

IL-12 p70

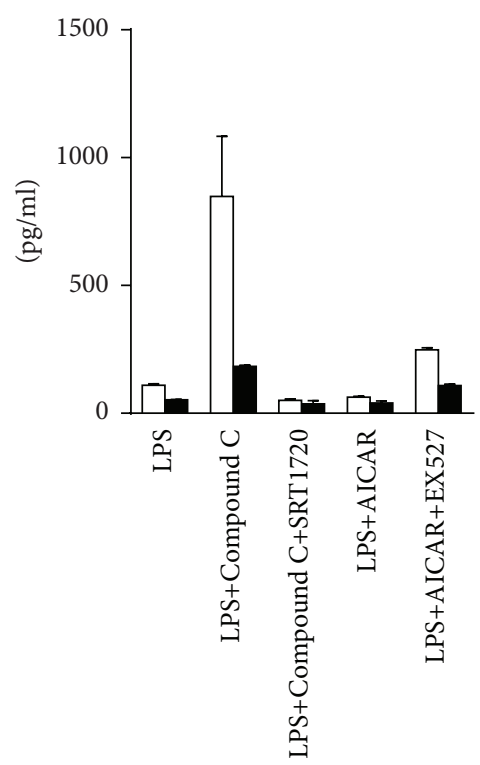

$\square$ IL-12 p40

(a)
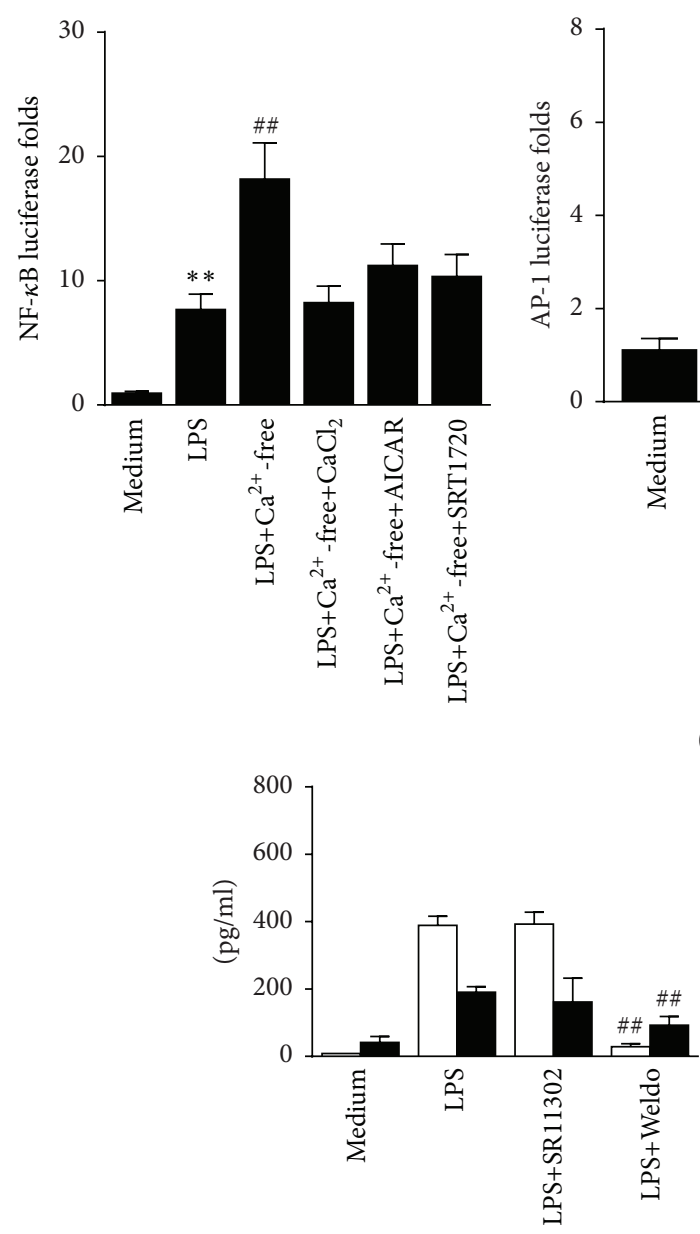

$\square$ IL-12 p40

IL-12 p70
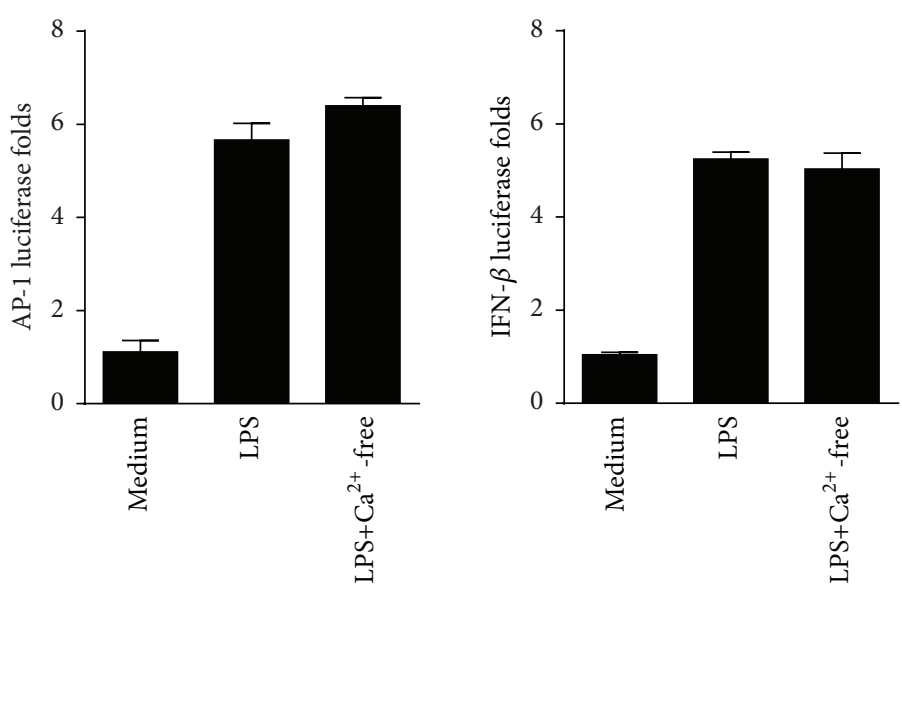

(b)

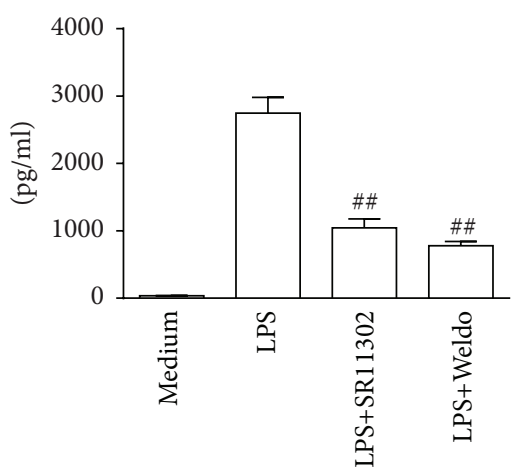

$\square$ IL-6

(c) 


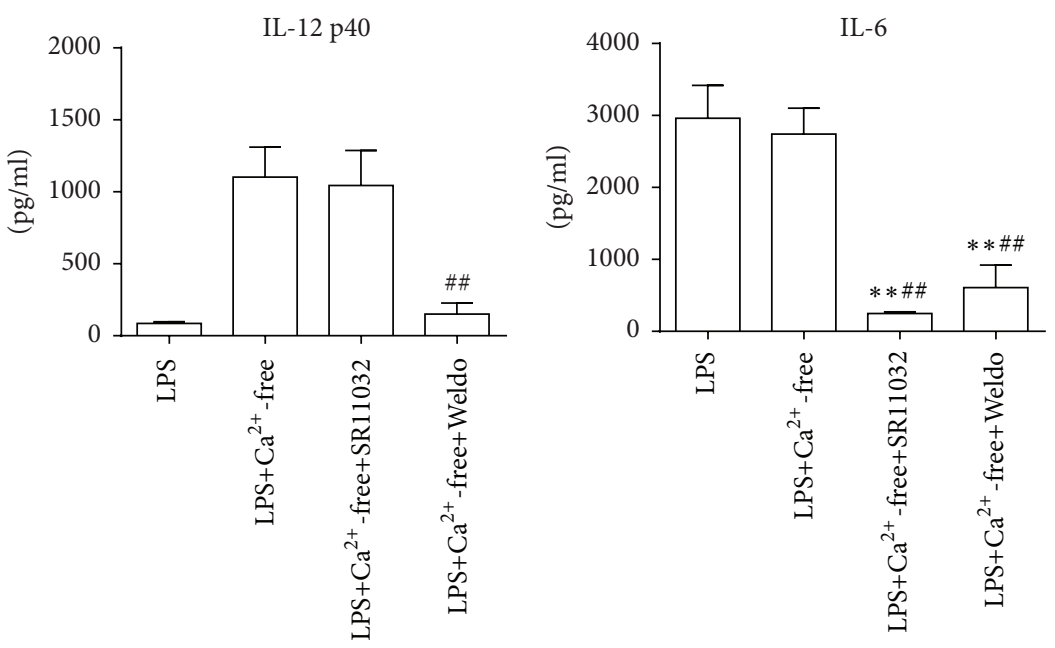

(d)

FIGURE 7: SIRT1 selectively attenuates transcription activity of NF- $\kappa$ B and suppresses IL-12 expression in LPS-stimulated RAW 264.7 cells. (a) Cells were treated with $1 \mu \mathrm{M}$ STO-609 alone or combined with $2 \mu \mathrm{M}$ SRT1720 or $1 \mathrm{mM}$ AICAR for $30 \mathrm{~min}$ before $100 \mathrm{ng} / \mathrm{mL}$ LPS stimulation (left). Cells were treated with $5 \mu \mathrm{M}$ Compound $\mathrm{C}$ alone or with $2 \mu \mathrm{M}$ SRT1720. Cells were also treated with $1 \mathrm{mM}$ AICAR alone or with $2 \mu \mathrm{M}$ EX527 (right). Supernatants were collected at $24 \mathrm{~h}$ and IL-12 p40/IL-12 p70 (c) and IL-6 (d) levels were detected by ELISA ( $n=3$ ). (b) Cells were transfected with plasmid pGL-luc2P/NF- $\kappa$ BRE for $24 \mathrm{~h}$ and further treated with $100 \mathrm{ng} / \mathrm{mL}$ LPS in normal DMEM, calcium-free DMEM, DMEM with $2 \mathrm{mM} \mathrm{CaCl}_{2}$, DMEM with $1 \mathrm{mM} \mathrm{AICAR,} \mathrm{or} \mathrm{DMEM} \mathrm{with} 2 \mu \mathrm{M}$ SRT1720 for $6 \mathrm{~h}$. Relative luciferase activity of NF- $\kappa \mathrm{B}$ against medium was presented. ${ }^{* *} P<0.01$ versus medium; ${ }^{\# \#} P<0.01$ versus LPS $(n=3)$. Cells were also transfected with plasmids pAP1-luc and pIRF3-luc for $24 \mathrm{~h}$ and further treated with $100 \mathrm{ng} / \mathrm{mL}$ LPS and LPS plus calcium-free DMEM for $6 \mathrm{~h}$. Relative luciferase activity of AP-1 and IRF3 was detected $(n=3)$. (c) Cells were treated with LPS alone with $5 \mu \mathrm{M}$ SR11302 or $10 \mu \mathrm{M}$ wedelolactone (Weldo) for $24 \mathrm{~h}$. Supernatant IL-12 p40/p70 and IL-6 levels were detected by ELISA. ${ }^{\#} P<0.01$ versus LPS $(n=3)$. (d) Cells were treated with LPS, LPS with calcium-free medium, or LPS with calcium-free medium alone with $5 \mu \mathrm{M}$ SR11302 or $10 \mu \mathrm{M}$ wedelolactone (Weldo) for $24 \mathrm{~h}$. Supernatant IL-12 p40/p70 and IL-6 levels were detected by ELISA. ${ }^{* *} P<0.01$ versus LPS; ${ }^{\# \#} P<0.01$ versus LPS plus calcium-free medium $(n=3)$.

for extracellular calcium influx across the plasma membrane. A number of direct channel blockers, including $\mathrm{La}^{3+}$, SKF96365, and 2-APB, were discovered and designed to inhibit SOCE $[30,31]$. In contrast, plasma membrane $\mathrm{Ca}^{2+}{ }_{-}$ ATPases use ATP to transport $\mathrm{Ca}^{2+}$. Therefore, ATP could enhance SOCE activity and facilitate calcium influx. The cytoplasmic calcium changes and calcium influx evoked by adenosine triphosphate (ATP) were widely observed in many different cell types [32]. Therefore, the SOCE based modulators are with opposite function in modulation calcium influx and thereby may facilitate the study of calcium-dependent regulation for IL-12.

CaMKK $\beta$ is a key signaling molecule activated by increased intracellular calcium. In immune cells, CaMKK $\beta$ expression is only found in monocyte/macrophage lineage and responsible for regulation of inflammation and immunity [14]. More recently, studies performed on myocytes and macrophages have reported that CaMKK $\beta$ phosphorylation could be induced by calcium influx or LPS stimulation [33]. In our study, we found that phosphorylation of CaMKK $\beta$ was induced by LPS stimulation and dependent on the increased calcium influx. To investigate the direct involvement of CaMKK $\beta$ in regulation of IL-12 production, we used its specific chemical inhibitor (STO-609) and siRNA to interfere with CaMKK $\beta$ activation and observed the marked increase of IL-12 release in LPS-stimulated murine macrophages [34]. The precise regulation patterns of CaMKK $\beta$ on inflammation remain largely unclear. In some cases, it was demonstrated that $\mathrm{CaMKK} \beta$ was protective against neuroinflammation by activating AMPK [35]. However, other authors reported that CaMKK $\beta$ was essentially involved in macrophage-mediated release of inflammatory cytokines [14]. Our finding demonstrated for the first time that CaMKK $\beta$ activation selectively suppressed LPS induced IL-12 production in murine macrophages. Therefore, the nature of CaMKK $\beta$ based regulation on inflammation and immunity still requires further study.

Whereas AMPK is a crucial sensor for energy balance, it has been also demonstrated that AMPK activation exerts significant anti-inflammatory and immunosuppressive effects on a variety of cell types. Recently, AMPK has been identified as a direct substrate of CaMKK $\beta$ for inflammation modulation in macrophages and this is currently accepted molecular pattern that explains the anti-inflammatory efficacy of CaMKK $\beta$ [36]. The enhanced AMPK $\alpha$ phosphorylation could be achieved by CaMKK $\beta$ activation with or without LPS $[35,36]$. However, we demonstrated that LPS-stimulated AMPK activation depends on calcium influx and CaMKK $\beta$ activation. Moreover, AMPK activation was necessary for calcium-dependent inhibition of LPS induced IL-12 production. Firstly, AMPK activation by AICAR further inhibited the suppression of IL-12 production, while inhibition of AMPK by Compound $\mathrm{C}$ resulted in a significant elevation of IL-12 release [36]. In AMPK $\alpha$ knockdown macrophages, elevated IL-12 levels were also detected upon LPS stimulation. Secondly, the increase of IL-12 production due to calcium 
depletion was abrogated when AICAR was given simultaneously. So these results support our presumption that AMPK is activated by the influx of calcium and activation of CaMKK $\beta$ to mediate IL-12 suppression.

The molecular mechanisms for AMPK are complicated. Emerging evidences demonstrated that AMPK can inhibit $\mathrm{NF}-\kappa \mathrm{B}$ activation and suppress the expression of inflammatory genes. AMPK could also modulate its downstream targets including SIRT1, PGC-l $\alpha$, p53, and FoxO3a, either directly or indirectly [37]. Recently, AMPK has been verified in coordination with another metabolic sensor, $\mathrm{NAD}^{+}$dependent type III deacetylase SIRT1, to regulate energy metabolism and inflammation $[18,38]$. AMPK regulates SIRT1 by increasing the $\mathrm{NAD}^{+} / \mathrm{NADH}$ ratio, which acts as a key upstream regulatory event that modulates the activity of SIRT1 and could be achieved by LPS induced TNF- $\alpha$ release in proinflammatory macrophages [38, 39]. SIRT1 expression could be modulated by LPS stimulation in macrophages/monocytes and upregulated SIRT1 expression was observed in resveratrol treated endothelial cells via the PRKA-AMPK pathway [40, 41]. Therefore, AMPK may act as an essential modulator for SIRT1 expression and we mainly focus on AMPK induced $\mathrm{NAD}^{+}$elevation and SIRT1 activation in this study.

In a previous study, an enhancement of SIRT1 activity in response to zymosan stimuli was demonstrated to reduce transcriptional activation of IL-12 in dendritic cells [42]. In our study, we identified that LPS induced the increase of $\mathrm{NAD}^{+} / \mathrm{NADH}$ ratio in murine macrophages and upregulated the expression of SIRT1, both indicating the elevated activity of SIRT1. Similar trends were found after AMPK activation by AICAR. We also verified the requirement of calcium in facilitating LPS induced SIRT1 activation which was abolished in medium without calcium. This presumption was further supported by our findings that selective activation of SIRT1 (SRT1720) activity suppressed IL-12 production but selective inhibition of SIRT1 (EX527) increased IL-12 levels upon LPS treatment $[43,44]$. Moreover, the increased IL-12 production due to calcium absence was abolished by SIRT1 activation.

To further clarify the regulatory patterns of CaMKKbAMPK-SIRT1 signaling cascade, we used combined manipulation of agonist and antagonist for CaMKKbeta-AMPK and SIRT1. Results demonstrated that CaMKK $\beta$ couples with AMPK and SIRT1 to mediate transcriptional inhibition on IL-12 production induced by LPS. To further establish the CaMKKb-AMPK-SIRT1 signaling cascade activation which further mediates the inhibition of NF- $\kappa \mathrm{B}$ activation by LPS, SIRT1 could negatively regulate inflammation by interfering with transcription events mediated by RelA/p65, PGC-1 $\alpha$, and PPAR $\alpha$ [18]. It has also been found that the activity of SIRT1 is closely related to NF- $\kappa$ B complex in controlling the resolution of inflammation [17]. In our study, depletion of extracellular calcium facilitated activation of NF- $\kappa \mathrm{B}$, which was abolished by the presence of $\mathrm{CaCl}_{2}$, AMPK activation (AICAR), or SIRT1 activation (SRT1720). In contrast, the activation of IRF3 or AP-1 was not affected in either group as compared with LPS alone. In our study, the I $\kappa \mathrm{B}-\alpha$ based $\mathrm{NF}-\kappa \mathrm{B}$ inhibitor wedelolactone [45] effectively attenuated
IL-6 and IL-12 production in RAW 264.7 cells. The AP-1 inhibitor SR11302 [46] only attenuated IL-6 production while it did not affect IL-12 secretion, although there exist different interpretations for IL-12 production mediated by AP-1 as well as its upstream kinases such as ERK1/2, p38, and JNK. Our results indicated that NF- $\kappa \mathrm{B}$ may be more importantly required for IL-12 p40 production, especially in the case that calcium influx was inhibited. However, further study is still necessary to determine transcriptional details involved in the ultimate regulation of IL-12 transcription by calciumCaMKK $\beta$-AMPK-SIRT1 pathway.

\section{Conclusion}

We demonstrate in this study that LPS induced production of IL-12 is affected by the increased intracellular calcium derived from extracellular influx. Such effects were mediated by activation of CaMKK $\beta, \mathrm{AMPK} \alpha$, and SIRT1 which ultimately lead to the suppression of NF- $\kappa$ B and downregulation of IL-12 production. Depletion of calcium influx inhibits those signaling events and thus increases LPS induced IL-12 production. Our findings discover a new connection between calcium, cellular energy metabolism, and inflammation, which may provide therapeutic targets for the precise regulation of inflammatory disorders.

\section{Competing Interests}

The authors declare that there is no conflict of interests.

\section{Acknowledgments}

This work is supported by grants from National Natural Science Foundation of China (81202566) and from Frontier and Applied Basic Research Program of Chongqing (cstc2014jcyjA10068).

\section{References}

[1] D. A. A. Vignali and V. K. Kuchroo, "IL-12 family cytokines: immunological playmakers," Nature Immunology, vol. 13, no. 8, pp. 722-728, 2012.

[2] R. Grumont, H. Hochrein, M. O'Keeffe et al., "c-Rel regulates interleukin 12 p70 expression in $\mathrm{CD}^{+}$dendritic cells by specifically inducing p35 gene transcription," The Journal of Experimental Medicine, vol. 194, no. 8, pp. 1021-1032, 2001.

[3] T. L. Murphy, M. G. Cleveland, P. Kulesza, J. Magram, and K. M. Murphy, "Regulation of interleukin 12 p40 expression through an NF- $\kappa$ B half-site," Molecular and Cellular Biology, vol. 15, no. 10, pp. 5258-5267, 1995.

[4] M. Utsugi, K. Dobashi, T. Ishizuka et al., "c-Jun N-terminal kinase negatively regulates lipopolysaccharide-induced IL-12 production in human macrophages: role of mitogen-activated protein kinase in glutathione redox regulation of IL-12 production," The Journal of Immunology, vol. 171, no. 2, pp. 628-635, 2003.

[5] T. Scharton-Kersten, C. Contursi, A. Masumi, A. Sher, and K. Ozato, "Interferon consensus sequence binding protein-deficient mice display impaired resistance to intracellular infection 
due to a primary defect in interleukin 12 p40 induction," Journal of Experimental Medicine, vol. 186, no. 9, pp. 1523-1534, 1997.

[6] S. Sanjabi, A. Hoffmann, H.-C. Liou, D. Baltimore, and S. T. Smale, "Selective requirement for c-Rel during IL-12 P40 gene induction macrophages," Proceedings of the National Academy of Sciences of the United States of America, vol. 97, no. 23, pp. 12705-12710, 2000.

[7] D. E. Clapham, “Calcium signaling," Cell, vol. 131, no. 6, pp. 1047-1058, 2007.

[8] G.-S. Lee, N. Subramanian, A. I. Kim et al., "The calciumsensing receptor regulates the NLRP3 inflammasome through $\mathrm{Ca}^{2+}$ and cAMP," Nature, vol. 492, no. 7427, pp. 123-127, 2012.

[9] S. Z. Vogel, S. Schlickeiser, K. Jürchott et al., “TCAIM decreases $\mathrm{T}$ cell priming capacity of dendritic cells by inhibiting TLRinduced $\mathrm{Ca}^{2+}$ influx and IL-2 production," Journal of Immunology, vol. 194, no. 7, pp. 3136-3146, 2015.

[10] B.-C. Chen, S.-L. Hsieh, and W.-W. Lin, "Involvement of protein kinases in the potentiation of lipopolysaccharide-induced inflammatory mediator formation by thapsigargin in peritoneal macrophages," Journal of Leukocyte Biology, vol. 69, no. 2, pp. 280-288, 2001.

[11] O. Letari, S. Nicosia, C. Chiavaroli, P. Vacher, and W. Schlegel, "Activation by bacterial lipopolysaccharide causes changes in the cytosolic free calcium concentration in single peritoneal macrophages," The Journal of Immunology, vol. 147, no. 3, pp. 980-983, 1991.

[12] L. Racioppi, P. K. Noeldner, F. Lin, S. Arvai, and A. R. Means, "Calcium/calmodulin-dependent protein kinase kinase 2 regulates macrophage-mediated inflammatory responses," The Journal of Biological Chemistry, vol. 287, no. 14, pp. 11579-11591, 2012.

[13] F. S. Sutterwala, G. J. Noel, R. Clynes, and D. M. Mosser, "Selective suppression of interleukin-12 induction after macrophage receptor ligation," The Journal of Experimental Medicine, vol. 185, no. 11, pp. 1977-1985, 1997.

[14] Y. Wang, Y. Yang, X. Liu et al., "Inhibition of clathrin/dynamindependent internalization interferes with LPS-mediated TRAM-TRIF-dependent signaling pathway," Cellular Immunology, vol. 274, no. 1-2, pp. 121-129, 2012.

[15] D. G. Hardie, F. A. Ross, and S. A. Hawley, "AMPK: a nutrient and energy sensor that maintains energy homeostasis," Nature Reviews Molecular Cell Biology, vol. 13, no. 4, pp. 251-262, 2012.

[16] L. A. J. O’Neill and D. Grahame Hardie, "Metabolism of inflammation limited by AMPK and pseudo-starvation," Nature, vol. 493, no. 7432, pp. 346-355, 2013.

[17] A. Kauppinen, T. Suuronen, J. Ojala, K. Kaarniranta, and A. Salminen, "Antagonistic crosstalk between NF- $\kappa$ B and SIRT1 in the regulation of inflammation and metabolic disorders," Cellular Signalling, vol. 25, no. 10, pp. 1939-1948, 2013.

[18] F. Yeung, J. E. Hoberg, C. S. Ramsey et al., "Modulation of NF- $\kappa$ B-dependent transcription and cell survival by the SIRT1 deacetylase," The EMBO Journal, vol. 23, no. 12, pp. 2369-2380, 2004.

[19] H. Yang, W. Zhang, H. Pan et al., "SIRT1 activators suppress inflammatory responses through promotion of p65 deacetylation and inhibition of NF- $\kappa$ B activity," PLoS ONE, vol. 7, no. 9, article e46364, 2012.

[20] W. S. Yang, Y. C. Park, J. H. Kim et al., "Nanostructured, selfassembling peptide $\mathrm{K} 5$ blocks TNF- $\alpha$ and $\mathrm{PGE}_{2}$ production by suppression of the AP-1/p38 pathway," Mediators of Inflammation, vol. 2012, Article ID 489810, 8 pages, 2012.
[21] N. Ramirez-Alejo and L. Santos-Argumedo, "Innate defects of the IL-12/IFN- $\gamma$ axis in susceptibility to infections by mycobacteria and Salmonella," Journal of Interferon and Cytokine Research, vol. 34, no. 5, pp. 307-317, 2014.

[22] H. Weighardt, C.-D. Heidecke, A. Westerholt et al., "Impaired monocyte IL-12 production before surgery as a predictive factor for the lethal outcome of postoperative sepsis," Annals of Surgery, vol. 235, no. 4, pp. 560-567, 2002.

[23] I. Munitic, M. L. Giardino Torchia, N. P. Meena, G. Zhu, C. C. Li, and J. D. Ashwell, "Optineurin insufficiency impairs IRF3 but not NF- $\kappa \mathrm{B}$ activation in immune cells," The Journal of Immunology, vol. 191, no. 12, pp. 6231-6240, 2013.

[24] L. R. Peters and M. Raghavan, "Endoplasmic reticulum calcium depletion impacts chaperone secretion, innate immunity, and phagocytic uptake of cells," Journal of Immunology, vol. 187, no. 2, pp. 919-931, 2011.

[25] S. Boubali, K. Liopeta, L. Virgilio et al., "Calcium/calmodulindependent protein kinase II regulates IL-10 production by human T lymphocytes: a distinct target in the calcium dependent pathway," Molecular Immunology, vol. 52, no. 2, pp. 51-60, 2012.

[26] L. Wang, I. Tassiulas, K.-H. Park-Min et al., “'Tuning' of type I interferon-induced Jak-STAT1 signaling by calcium-dependent kinases in macrophages," Nature Immunology, vol. 9, no. 2, pp. 186-193, 2008.

[27] R. J. Francis, R. E. Butler, and G. R. Stewart, "Mycobacterium tuberculosis ESAT-6 is a leukocidin causing $\mathrm{Ca}^{2+}$ influx, necrosis and neutrophil extracellular trap formation," Cell Death and Disease, vol. 5, no. 10, Article ID e1474, 2014.

[28] S. Bertin, Y. Aoki-Nonaka, P. R. de Jong et al., "The ion channel TRPV1 regulates the activation and proinflammatory properties of CD $4^{+}$T cells," Nature Immunology, vol. 15, no. 11, pp. 10551063, 2014.

[29] V. N. Tomilin, A. L. Cherezova, Y. A. Negulyaev, and S. B. Semenova, "TRPV5/V6 channels mediate $\mathrm{Ca}^{2+}$ influx in jurkat T cells under the control of extracellular $\mathrm{pH}$," Journal of Cellular Biochemistry, vol. 117, no. 1, pp. 197-206, 2016.

[30] A. Gallois, J.-L. Bueb, and E. Tschirhart, "Effect of SK\&F 96365 on extracellular $\mathrm{Ca}^{2+}$-dependent $\mathrm{O}_{2}^{-}$production in neutrophillike HL-60 cells," European Journal of Pharmacology, vol. 361, no. 2-3, pp. 293-298, 1998.

[31] J. W. Putney, "Pharmacology of store-operated calcium channels," Molecular Interventions, vol. 10, no. 4, pp. 209-218, 2010.

[32] K. Pászty, A. J. Caride, Ž. Bajzer et al., "Plasma membrane $\mathrm{Ca}^{2+}$ ATPases can shape the pattern of $\mathrm{Ca}^{2+}$ transients induced by store-operated $\mathrm{Ca}^{2+}$ entry," Science Signaling, vol. 8, no. 364, article ra19, 2015.

[33] T. S. Mathew, R. K. Ferris, R. M. Downs, S. T. Kinsey, and B. L. Baumgarner, "Caffeine promotes autophagy in skeletal muscle cells by increasing the calcium-dependent activation of AMPactivated protein kinase," Biochemical and Biophysical Research Communications, vol. 453, no. 3, pp. 411-418, 2014.

[34] H. Tokumitsu, H. Inuzuka, Y. Ishikawa, M. Ikeda, I. Saji, and R. Kobayashi, "STO-609, a specific inhibitor of the $\mathrm{CA}^{2+} /$ calmodulin-dependent protein kinase kinase," Journal of Biological Chemistry, vol. 277, no. 18, pp. 15813-15818, 2002.

[35] X. Zhou, Y. Cao, G. Ao et al., "CaMKK $\beta$-dependent activation of AMP-activated protein kinase is critical to suppressive effects of hydrogen sulfide on neuroinflammation," Antioxidants \& Redox Signaling, vol. 21, no. 12, pp. 1741-1758, 2014.

[36] I. P. Salt and T. M. Palmer, "Exploiting the anti-inflammatory effects of AMP-activated protein kinase activation," Expert 
Opinion on Investigational Drugs, vol. 21, no. 8, pp. 1155-1167, 2012.

[37] R. Mounier, M. Théret, L. Arnold et al., "AMPK $\alpha 1$ regulates macrophage skewing at the time of resolution of inflammation during skeletal muscle regeneration," Cell Metabolism, vol. 18, no. 2, pp. 251-264, 2013.

[38] M. Scheibye-Knudsen, S. J. Mitchell, E. F. Fang et al., "A highfat diet and $\mathrm{NAD}^{+}$activate sirtl to rescue premature aging in cockayne syndrome," Cell Metabolism, vol. 20, no. 5, pp. 840855, 2014.

[39] A. J. Al-Shabany, A. J. Moody, A. D. Foey, and R. A. Billington, "Intracellular NAD+ levels are associated with LPS-induced TNF-alpha release in pro-inflammatory macrophages," Bioscience Reports, vol. 36, no. 1, pp. e00301-e00301, 2016.

[40] T. F. Liu, V. T. Vachharajani, B. K. Yoza, and C. E. McCall, "NAD" ${ }^{+}$-dependent sirtuin 1 and 6 proteins coordinate a switch from glucose to fatty acid oxidation during the acute inflammatory response," The Journal of Biological Chemistry, vol. 287, no. 31, pp. 25758-25769, 2012.

[41] M.-L. Chen, L. Yi, X. Jin et al., "Resveratrol attenuates vascular endothelial inflammation by inducing autophagy through the cAMP signaling pathway," Autophagy, vol. 9, no. 12, pp. 20332045, 2013.

[42] Y. Alvarez, M. Rodríguez, C. Municio et al., "Sirtuin 1 is a key regulator of the interleukin-12 p70/interleukin-23 balance in human dendritic cells," The Journal of Biological Chemistry, vol. 287, no. 42, pp. 35689-35701, 2012.

[43] M. Gertz, F. Fischer, G. T. T. Nguyen et al., "Ex-527 inhibits Sirtuins by exploiting their unique $\mathrm{NAD}^{+}$-dependent deacetylation mechanism," Proceedings of the National Academy of Sciences of the United States of America, vol. 110, no. 30, pp. E2772-E2781, 2013.

[44] J. C. Milne, P. D. Lambert, S. Schenk et al., "Small molecule activators of SIRT1 as therapeutics for the treatment of type 2 diabetes," Nature, vol. 450, no. 7170, pp. 712-716, 2007.

[45] M. Kobori, Z. Yang, D. Gong et al., "Wedelolactone suppresses LPS-induced caspase-11 expression by directly inhibiting the IKK complex," Cell Death and Differentiation, vol. 11, no. 1, pp. 123-130, 2004.

[46] H. Kankaanranta, P. Ilmarinen, X. Zhang et al., "Tumour necrosis factor- $\alpha$ regulates human eosinophil apoptosis via ligation of TNF-receptor 1 and balance between NF- $\kappa$ B and AP1," PLoS ONE, vol. 9, no. 2, Article ID e90298, 2014. 


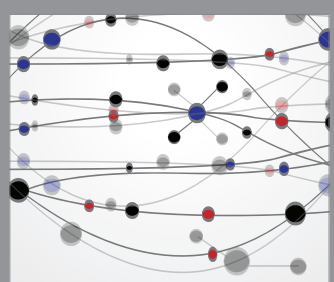

The Scientific World Journal
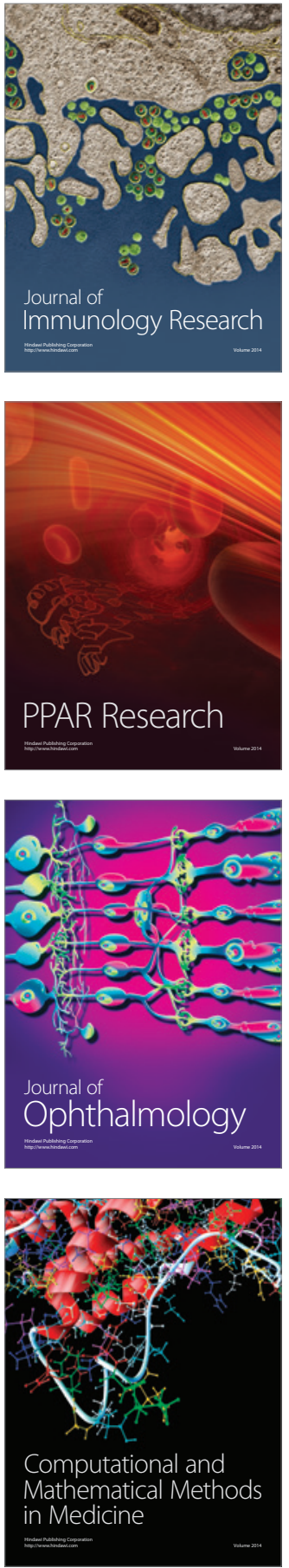

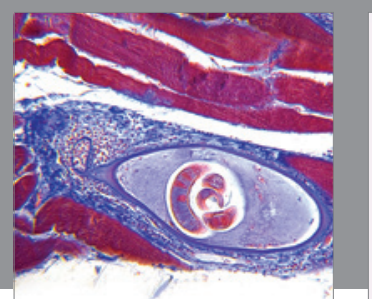

Gastroenterology Research and Practice

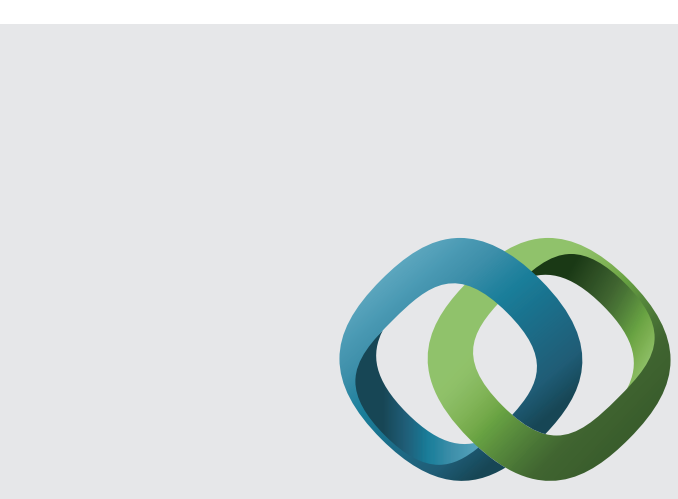

\section{Hindawi}

Submit your manuscripts at

http://www.hindawi.com
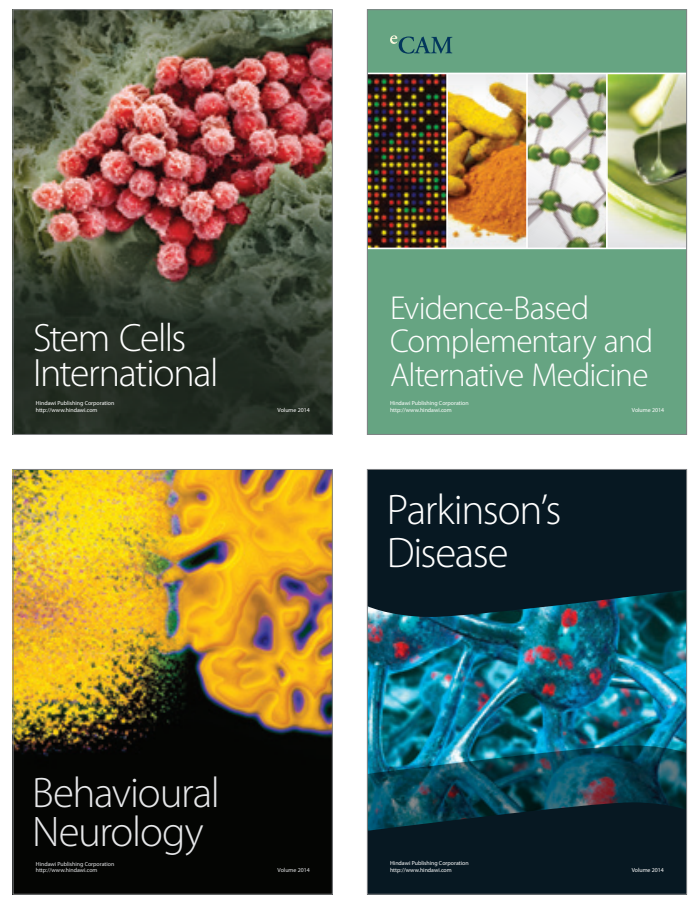
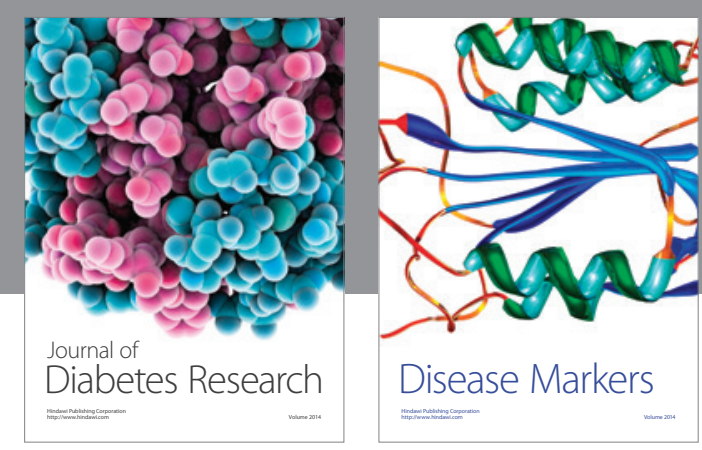

Disease Markers
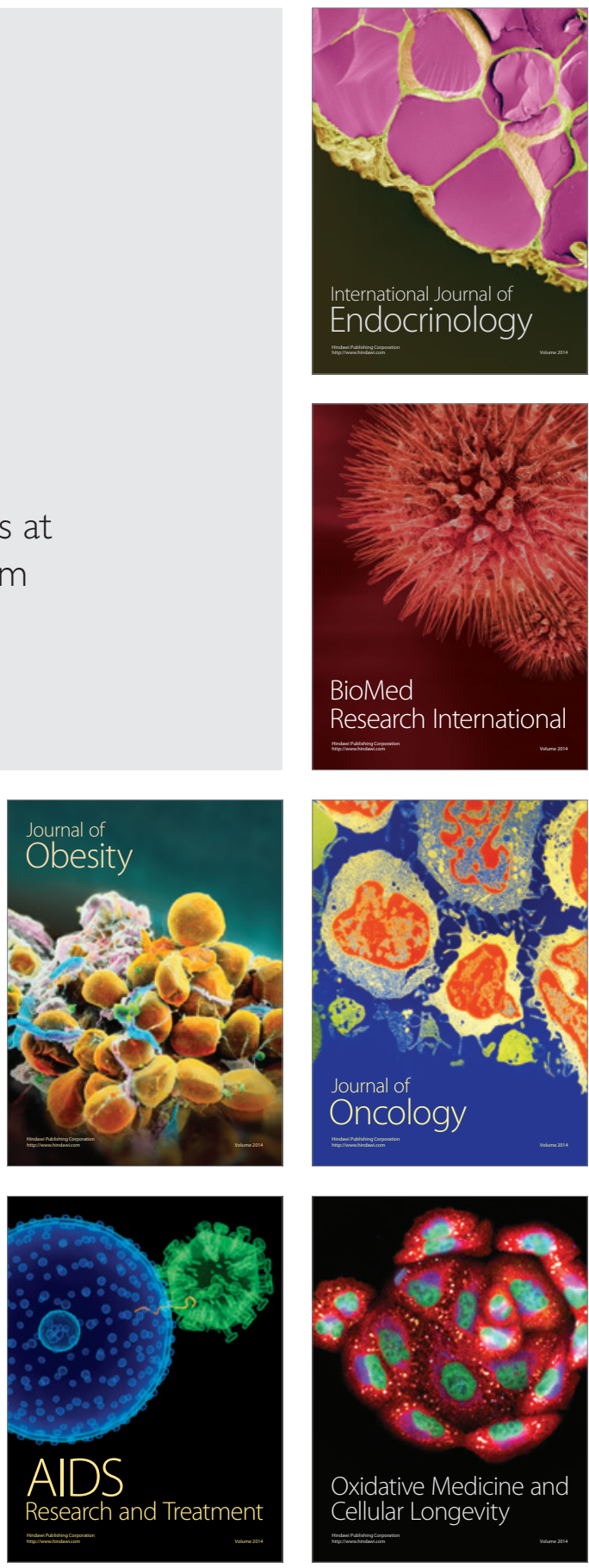